\title{
PROJECTIVE PLANES
}

\author{
BY \\ MARSHALL HALL
}

1. Introduction. The Theorem of Desargues has played a central role in the study of the foundations of projective geometry. Its validity has been shown to be equivalent to the possibility of the introduction of homogeneous coordinates from a skew-field, and also to the possibility of imbedding a plane in a space of higher dimension. A synthetic proof of the theorem, using a third dimension, is relatively easy, but two dimensional proofs failed and it was eventually realized that projective planes existed in which the theorem was not valid. Non-Desarguesian planes have received attention primarily as curious counter-examples, proving the necessity of assuming, along with the axioms of connection, either the existence of a third dimension, or axioms of congruence $\left({ }^{1}\right)$. But the full class of projective planes has a much better claim than this for attention. Projective planes are the logical basis for the investigation of combinatorial analysis, such topics as the Kirkman schoolgirl problem and the Steiner triple systems being interpretable directly as plane configurations, various problems in non-associative or nondistributive algebras, and the problems of universal identities in groups and skew-fields, as well as certain problems on structure (lattice) theory and the theory of partially ordered $\operatorname{sets}\left({ }^{2}\right)$.

The author has made an attempt to investigate projective planes as systematically as possible. A considerable portion of these investigations is not included here either because of the incomplete and unsatisfactory nature of the results or because of lack of generality. War duties have forced the postponement of the completion of this work. The investigations here proceed along two main lines. $\S \S 2,3$, and 4 are concerned with methods of constructing the most general projective planes, and demonstrating the extent of variety which exists in the class of projective planes. In $\$ 4$, the free planes, comparable in certain ways to free groups, are studied. Free planes, generated by finite configurations, are characterized (Theorems 4.8 and 4.13) and a

Presented to the Society, September 10, 1940; received by the editors October 22, 1942.

(1) Certainly this is Hilbert's attitude. See D. Hilbert [1]. This reference and all others in the paper are to the bibliography at the end of the paper.

(2) In a recent Colloquium publication it was said "The study of plane projective geometries takes us into one of the most mysterious and fascinating parts of combinatory analysis-a subject concerning which little is known." On the Kirkman problem, see T. P. Kirkman [1], E. W. Davis [1], and the bibliography on the problem, O. Eckenstein [1]. For triple systems, see A. Emch [1]. Geometric algebras related to quantum theory are investigated in P. Jordan, J. von Neumann, and E. Wigner [1]. F. Levi [1] applies the theory of configurations to combinatorial topology. 
canonical set (Theorem 4.12) is determined. As a corollary it follows that the subplanes of a free plane are free planes. This analogy with free groups does not seem to go so far as to enable us to consider an arbitrary plane as a homomorphic image of a free plane. Certainly this cannot be done by a lattice homomorphism, and no clearly defined homomorphism of partial ordering is determined. If, as a consequence of identifications, we find two different points lying on two different lines, we must identify the points or identify the lines, but there is no clear reason for preferring one identification to the other. This fundamental difficulty has no analogue in the corresponding problem for groups.

$\S \S 5$ and 6 proceed in a direction different from, but in a sense complementary to, the direction of the earlier sections. The earlier sections are concerned mainly with the geometry of open configurations, the later ones with the geometry of confined configurations. (The definitions of these two terms immediately precede and follow Theorem 4.8.) An effort is made to provide an algebraic substitute in the general plane for the coordinatizing skew-field in Desarguesian planes. Two related systems of coordinates are introduced, one a system of permutations (essentially projections), the other a ring defined by a ternary operation. It is perhaps unfortunate that the permutations force two points into a special role and that the ternary ring places a single quadrilateral in a special role. Theorems on configurations are associated with algebraic relations in these systems. The algebraic consequences of these relations are of great importance in characterizing the geometric conclusions to be drawn, and enable us to determine the geometric dependence or independence of configuration theorems. Many algebraic problems and new types of algebraic systems arise from natural geometric questions, and the fullest investigation of these is called for. The same geometric system (such as a web or a complete plane) may be associated with two or more non-isomorphic algebraic systems, each of which may be constructed from any other by certain rules. (See rule (5.12) in Theorem 5.9 and the examples in appendix II.) This geometric equivalence, stronger than algebraic isomorphism, is a valuable algebraic tool, comparable to the theory of classes of algebras.

2. The axioms of projective geometry: The projective plane. Hilbert $\left(^{3}\right)$ who was as much interested in metric geometry as in projective geometry bases his studies of the foundations of geometry on five sets of axioms: I. Axioms of connection, II. Axioms of order, III. Axioms of parallels, IV. Axioms of congruence, and V. Axioms of continuity. Of these only I and III define abstract Euclidean space and the introduction of ideal elements at infinity reduces this space to a projective space. Axioms II, IV, and particularly $\mathrm{V}$ restrict the coordinate skew-field (given by the von Staudt construction using I and III) to the real number field. Veblen and Young $\left({ }^{4}\right)$,

(3) D. Hilbert [1].

(4) O. Veblen and J. W. Young [1]. 
being concerned solely with projective geometry, proceed somewhat differently to the same end. More recently Garrett Birkhoff $\left(^{5}\right)$ has defined projective geometry as an irreducible complemented modular lattice (complemented Dedekind structure) and shows the equivalence of his axioms to those of Veblen and Young.

The principal result of these investigations is that the points of a projective space of three or more dimensions, which is necessarily Desarguesian, or a Desarguesian plane may be coordinatized by the von Staudt construction with elements from a skew-field, and the $(k-1)$-dimensional subspaces of a $k$-dimensional projective space contain those points which satisfy a linear equation. The skew-field will be a commutative field if and only if the Theorem of Pappus holds, while further conditions are required if it is wished to restrict the field to the field of real numbers.

Coming to the structure of projective spaces of less than three dimensions, the void space or a point can hardly be said to have any structure, while a line is completely determined by the number of points on it. This leaves only the non-Desarguesian planes, whose structure is non-trivial, to be characterized by algebraic means or otherwise.

Before proceeding to a study of projective planes it seems desirable to axiomatize the plane as simply as possible. The following axioms may be used:

P1. Any two distinct points are contained in one and only one line.

P2. Any two distinct lines contain one and only one point in common.

P3. There exist four points, no three of which are contained in one line.

The proof that these axioms define a plane in exactly the sense of Birkhoff or Veblen and Young is quite simple and will not be given here. P3 serves the purpose of axiom EO of Veblen and Young and might easily be replaced by a number of equivalent conditions. It seems more elegant to the author to require the existence of only four points of a specified kind rather than the infinity of conditions in axiom EO.

The removal of a line and the points on it from a projective plane $\pi$ leaves a Euclidean plane $\pi^{*}$ whose points and lines satisfy the following axioms:

EP1. Any two distinct points are contained in one and only one line.

EP2. Two distinct lines contain at most one point in common.

EP3. The plane contains four points, no three of which are contained in one line.

EP4. Through a point $P$ not contained in a line $L$ there is exactly one line $M$ which has no point in common with $L$.

From EP4 two lines which are parallel to (that is, do not intersect) a third line are parallel to each other. If to $\pi^{*}$ we add a point of intersection for

(s) Garrett Birkhoff [1]. Also K. Menger [1, 2]. These papers achieve independently virtually the same formulation. 
each system of parallels and then add a single line containing all these new points we obtain a projective plane $\pi$. Clearly the Euclidean plane $\pi^{*}$ determines $\pi$ uniquely.

We may include as planes the systems satisfying the axioms P1 and P2 but not P3. These are easily found to be

1. the void plane,

2. a plane containing a single point and no lines,

3. a plane containing a single line and no points,

4. a plane containing a single point and one or more lines through the point,

5. a plane containing a single line and one or more points on the line,

6. a plane containing a single line and a single point not on the line,

7. a plane containing a point $P$ on a line $L$ with one or more additional lines through $P$ and one or more additional points on $L$,

8. a plane containing a line $L$, and one or more points on $L$, a point $P$ not on $L$ and one or more lines through $P$ joining $P$ to the points of $L$.

These systems will be referred to as degenerate or improper planes. Note that every plane in which there are enough points on each line contains all these types of degenerate planes. In defining proper planes P3 may be replaced by any number of other axioms which exclude these degenerate cases.

Why is it that projective spaces of three or more dimensions are sufficiently regular to be coordinatized by skew-fields while projective nonDesarguesian planes exist which cannot be so coordinatized? One way of answering this fundamental question is the following: In a space configuration of three or more dimensions the identification of a line, constructed as the intersection of two planes, with a line, constructed as the union of two points forces the identification of further constructed elements and the establishment of non-trivial configurations such as the Desargues configuration, while in the plane a line may be constructed only as a union of two points, a point as the intersection of two lines and there are no forced identifications. Consider a proof of the Theorem of Desargues for the case in which the triangles are not coplanar. (See Fig. 1.)

Given: Triangles $A_{1} B_{1} C_{1}, A_{2} B_{2} C_{2}$, not in the same plane, and $A_{1} A_{2}, B_{1} B_{2}$, and $C_{1} C_{2}$ passing through the point $P$.

To prove: That $A_{1} B_{1}$ and $A_{2} B_{2}, A_{1} C_{1}$ and $A_{2} C_{2}, B_{1} C_{1}$ and $B_{2} C_{2}$ intersect in points $C_{3}, B_{3}$, and $A_{3}$ which are collinear.

Proof. $A_{1} B_{1}$ and $A_{2} B_{2}$ both lying in the plane $P A_{1} B_{1}$ must intersect in a point $C_{3}$. Similarly $A_{1} C_{1}$ and $A_{2} C_{2}$ intersect in a point $B_{3}$ and $B_{1} C_{1}$ and $B_{2} C_{2}$ intersect in a point $A_{3}$. But $A_{3}, B_{3}$, and $C_{3}$ must lie in both the plane of $A_{1} B_{1} C_{1}$ and the plane of $A_{2} B_{2} C_{2}$ and hence on $m$, the line of intersection of these two planes. Hence the lines $A_{3} B_{3}, A_{3} C_{3}$, and $B_{3} C_{3}$ must coincide with this intersection and the points $A_{3}, B_{3}$, and $C_{3}$ are collinear. 
Here the kernel of the proof lies in the identification of $A_{3} B_{3}, A_{3} C_{3}$, and $B_{3} C_{3}$ with $m$ and hence with each other. As we shall see in the next section there are no such forced identifications in the plane.

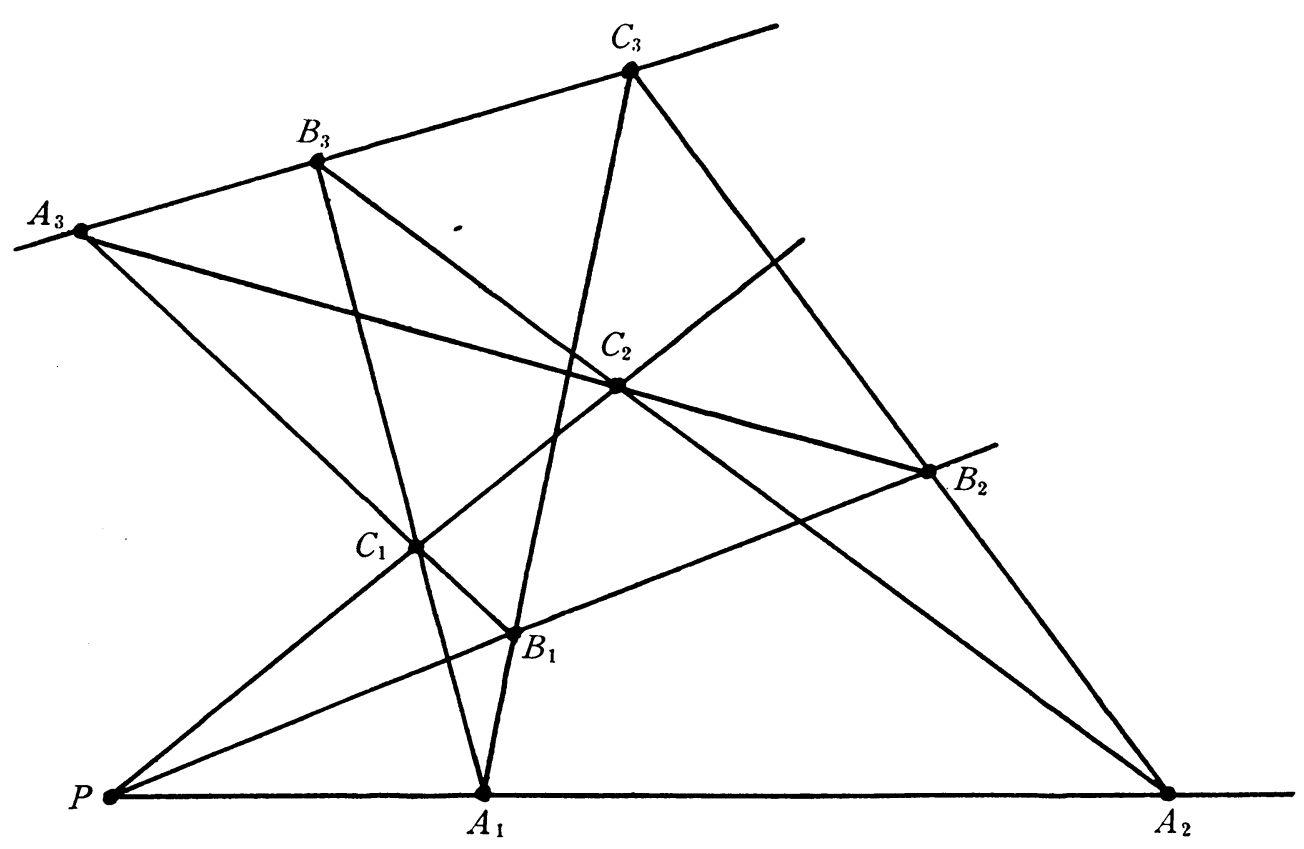

FIG. 1

3. The generation of projective planes. Given any set of points and lines in a plane, we may obtain further points and lines of the plane by constructing the lines joining points of the set and by finding the points of intersection of lines of the set. We may repeat this process on the enlarged set and continue as often as we please. All points and lines obtainable in this way are said to be generated by the given set. For example, all points on a line and two points not on the line generate the entire plane in four steps. But obviously the entire plane may be generated by the two points and only part of the points on the line or in various other ways. The rational plane (rational net in Veblen-Young) is generated by any quadrilateral in it.

We may use the method of generation to construct all possible projective planes.

Definition. A partial plane is any system of points and lines such that:

p1. There is at most one line through any two distinct points.

p2. There is at most one point common to two distinct lines. 
A partial plane is degenerate if it is contained in a degenerate plane. A partial plane is proper if it generates a proper plane. This it will do if and only if it contains one of the following:

1. four points, no three collinear,

2. four lines, no three copunctal,

3. three non-collinear points and a line not through any one of them,

4. three non-copunctal lines and a point not on any one of them,

5. a triangle of points and lines not sides through two of these,

6. a triangle of lines and points on two sides of it.

A representation of a partial plane $\pi$ is a table giving a list of the lines $\left(L_{1}, \cdots\right)$ of $\pi$ and listing after each line $L_{1}$ points $\left(A_{1}, \cdots\right)$ of $\pi$ contained in $L_{1}$. Such a representation is said to be explicit if it is to be understood that the points and lines are distinct as listed and that no point of $\pi$ lies on any line of $\pi$ unless it is so listed. We say that $\pi_{2}$ is complete in $\pi_{1}$ if $\pi_{1} \supset \pi_{2}$ and any point of intersection of lines of $\pi_{2}$ which lies in $\pi_{1}$ also lies in $\pi_{2}$ and if every line joining two points of $\pi_{2}$ which lies in $\pi_{1}$ also lies in $\pi_{2}$. A plane satisfies the axioms of a partial plane and may also be represented as a partial plane. A partial plane $\pi_{1}$ is said to be isomorphic to a partial plane $\pi_{2}$ if and only if there is a 1-1 correspondence between a representation $\left[L^{1}, A^{1}\right]$ of $\pi_{1}$ and $\left[L^{2}, A^{2}\right]$ of $\pi_{2}$ such that if $A^{1} C L^{1}$ and $L^{1} \rightleftarrows L^{2}, A^{1} \rightleftarrows A^{2}$ then $A^{2} C L^{2}$ and conversely. Isomorphic partial planes will not in general be considered distinct.

A representation of a partial plane is

\begin{tabular}{|c|c|c|}
\hline$L_{1}: \quad P A_{1} A_{2}$ & $L_{5}: \quad A_{2} B_{2} C_{3}$ & $L_{9}:$ \\
\hline$P B_{1} B_{2}$, & $A_{1} C_{1} B_{3}$, & $L_{10}:$ \\
\hline$P C_{1} C_{2}$ & $A_{2} C_{2} B_{3}$ & $L_{11}$ : \\
\hline$A_{1} B_{1} C_{3}$ & $L_{8}: \quad B_{1} C_{1} A_{3}$, & $L_{12}:$ \\
\hline
\end{tabular}

If $P_{1}, \cdots, P_{s}$ are points no two of which are joined in the representation of a partial plane, we may extend the representation formally by adding a line $L: P_{1}, \cdots, P_{s}$ to the representation, or if $L_{1}, \cdots, L_{r}$ are lines no two of which intersect, we may extend by adjoining a point $P$ to each of $L_{1}, \cdots, L_{r}$. The larger table is a representation of a partial plane which contains the original partial plane but which now contains a line $L$ joining $P_{1}, \cdots, P_{8}$ or in which $L_{1}, \cdots, L_{r}$ now intersect in $P$. This method of extension may be used to imbed any partial plane in a complete plane.

TheOREM 3.1. Given a partial plane $\pi_{1}$, and a representation $R_{1}$ of $\pi_{1}$. If $\pi_{1}$ is degenerate, then any plane with enough points on each line contains $\pi_{1}$ explicitly. If $\pi_{1}$ is nondegenerate, there is a complete plane $\pi$ generated by $\pi_{1}$ in which the representation of $\pi_{1}$ by $R_{1}$ is explicit.

Proof. It has already been noted above that a degenerate plane is con- 
tained in any plane with enough points on each line. The representation of a degenerate plane is necessarily explicit.

Given a nondegenerate partial plane $\pi_{1}$ and a representation $R_{1}$ of $\pi_{1}$. Form sets $S_{i}\left(P_{i 1}^{1}, \cdots, P_{i r}^{1}\right)$ of points $P^{1}$ of $\pi_{1}$ such that:

(1) Every pair of points $P^{1}, Q^{1}$ of $\pi_{1}$ not joined by a line of $\pi_{1}$ is in exactly one set $S$.

(2) No pair of points $P^{1}, Q^{1}$ in a set $S$ is joined by a line of $\pi_{1}$.

We may certainly define such sets in at least one way. We may take each set $S$ with only two points in it, and include a point pair $P, Q$ as a set if and only if $P$ and $Q$ are not joined in $\pi_{1}$. We now define a partial plane $\pi_{2}$ by a representation $R_{2}$ in which there are points $P^{1}$ and lines $L^{0}, L^{2} . P^{1}$ and $L^{0}$ are the points and lines of $R_{1}$ and $P^{1} C L^{0}$ in $R_{2}$ if and only if $P^{1} C L^{0}$ in $R_{1}$. For each set $S_{i}\left(P_{i 1}^{1}, \cdots, P_{i r}\right)$ we define a line $L_{i}^{2}$ in $R_{2}$ and say that $P_{i j}^{1} \subset L_{i}^{2}$, $j=1, \cdots, r$, and $P^{1} \nsubseteq L_{i}^{2}$ otherwise. Then $R_{2}$ is a representation of a partial plane $\pi_{2}$ containing $\pi_{1}$ explicitly. Every pair of points in $\pi_{1}$ is joined by a line in $\pi_{2}$ if not already joined in $\pi_{1}$. Similarly we may extend $R_{2}$ to a representation $R_{3}$ of a partial plane $\pi_{3}$ which contains $\pi_{2}$ explicitly and such that every pair of lines in $\pi_{2}$ intersects in a point of $\pi_{3}$ if the pair does not already intersect in a point of $\pi_{2}$.

We may continue this process a denumerable number of times. For each $R_{2 n}$ of a $\pi_{2 n}$ we form sets of two or more nonintersecting lines as was done for $R_{\mathbf{2}}$ and use these sets to define new intersection points in a representation $R_{2 n+1}$ of a $\pi_{2 n+1}$ containing $\pi_{2 r}$ explicitly. For each $R_{2 n+1}$ of a $\pi_{2 n+1}$ we form sets of two or more points which are not collinear in $R_{2 n+1}$ and use these sets to define new lines in a representation $R_{2 n+2}$ containing $R_{2 n+1}$ explicitly. Every incidence of points and lines in a representation $R_{i}$ will occur in a representation $R_{j}, j>i$, and no incidences of points and lines of $R_{i}$ will occur in $R_{j}$, $j>i$, except those already in $R_{i}$. Requirement (1) of our extensions assures us that we do not adjoin two new intersection points to a pair of lines, or two new lines through a pair of points. Requirement (2) assures us that we do not adjcin a new intersection point for lines which already intersect or a new line through points already joined. Hence every $R_{n}$ is a partial flane.

Now define a representation $R$ of points and lines $P, L$ occurring in any $R_{n}, n=1,2, \cdots$, and let $P^{i} C L^{j}$ in $R$ if and only if $P^{i} C L^{j}$ in some $R_{n}$. This determination of incidence is unambiguous, for it is determined in $R_{\max (i, j)}$, the first $R_{n}$ containing both $P^{i}$ and $L^{j}$, and is not altered in any succeeding $R_{n}$. Hence $R$ contains $R_{1}$ explicitly, and satisfies the requirements for a partial plane. Every pair of lines of $R_{2 s}$ has an intersection either in $R_{28}$ or in $R_{28+1}$, and every pair of points in $R_{2 s+1}$ is joined by either a line of $R_{2 s+1}$ or by a line of $R_{2 s+2}$. Hence $R$ is not only a partial plane but is a complete plane.

It is in the sense of this theorem that it was said in $\$ 2$ that there are no forced identifications in projective planes. For example, (3.1) is a representa- 
tion of a partial plane which may be extended to a complete plane without forcing the identification of $L_{10}, L_{11}$, and $L_{12}$ as would be necessary in any Desarguesian projective space.

The consequences of this theorem are numercus when the very general nature of a partial plane is taken into account. Let us call a subplane $\pi^{\prime}$ of a plane $\pi$ a set of points and lines which satisfy P1, 2, 3 or merely P1, 2. Now we may take as a partial plane any set of disjoint planes and generate a plane containing these as subplanes.

TheOREM 3.2. An arbitrary set of planes may occur among the subplanes of a plane.

The subplanes of a plane form a structure-lattice if we take the crosscut $\pi_{1} \cap \pi_{2}$ of subplanes $\pi_{1}, \pi_{2}$ to be the points and lines common to both subplanes and the union $\pi_{1} \cup \pi_{2}$ to be the subplane generated by $\pi_{1}$ and $\pi_{2}$. Here it is necessary to include degenerate subplanes. Unfortunately the structure-lattice of subplanes need not be particularly regular. At least it is very easy to construct a plane in which this structure-lattice is not a Dedekind structure (modular lattice). Let us take a 7 -point plane $\pi_{1}$ and a 13 -point plane $\pi_{2}$ with no points or lines in common and let a line of $\pi_{1}$ intersect a line of $\pi_{2}$ in a point $P$. Now construct a 21 -point plane $\pi_{3}$ generated by $\pi_{1}$ and $P$ and let the partial plane of $\pi_{2}$ and $\pi_{3}$ be extended to a complete plane $\pi$ (necessarily larger than $\pi_{3}$ ). We now have the following diagram from the structurelattice. Here $\pi_{3} \supset \pi_{1}$ and $\pi_{3}=\pi_{3} \cap\left(\pi_{2} \cup \pi_{1}\right) \neq\left(\pi_{3} \cap \pi_{2}\right) \cup \pi_{1}=\pi_{1}$, and the subplane structure-lattice is certainly not modular.

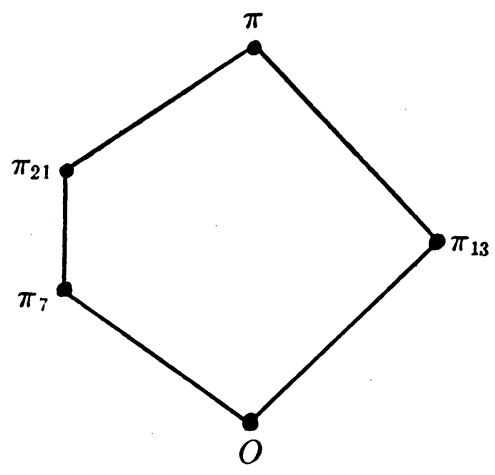

FIG. 2

The great generality of projective planes has so far been demonstrated in showing that (1) the configurations in different parts are independent and (2) the structure-lattice of subplanes is not greatly restricted, at least in any familiar way.

4. Free extensions and free planes. The simplest way of extending a par- 
tial plane is to let each new point adjoined be the intersection of only two old lines and each new line join only two old points. Such an extension will be called a free extension.

From the method of constructing free extensions we have immediately the following theorem:

THEOREM 4.1. The free extension of a partial plane to a complete plane is unique.

For two free extensions of a partial plane to a complete plane can differ only in the order in which new elements are added. Every new element is constructed from points and lines of the given partial plane in a single way.

THEOREM 4.2. If $\pi_{2}$ is a free extension of $\pi_{1}$ and $\pi_{3}$ is a free extension of $\pi_{2}$, then $\pi_{3}$ is a free extension of $\pi_{1}$. The free extension of $\pi_{2}$ to a complete plane is the free extension of $\pi_{1}$ to a complete plane.

THEOREM 4.3. If $\pi_{1} \supset \pi_{2}$ and $\pi_{2}$ is complete in $\pi_{1}$ then the free extension $\pi_{1}^{\prime}$ of $\pi_{1}$ contains as a subplane the free extension $\pi_{2}^{\prime}$ of $\pi_{2}$. If $\pi_{2}$ is a proper part of $\pi_{1}$, then $\pi_{2}$ is a proper part of $\pi_{1}^{\prime}$.

Since $\pi_{2}$ is complete in $\pi_{1}$ its extension will include only new elements.

We may call the free extension of a partial plane $\pi_{1}$ to a complete plane simply the free extension of $\pi_{1}$. The free extension of $n-2$ points on a line and 2 points not on it will be written $\pi^{n}$.

THEOREM 4.4. The free extension of any non-degenerate partial plane $\pi_{1}$ which is not a complete plane contains $a \pi^{4}$ as a subplane.

Proof. Since $\pi_{1}$ is not a complete plane and is nondegenerate, it contains either (a) a pair of points $P_{1}, P_{2}$ not joined by any line or (b) a pair of lines $L_{1}, L_{2}$ which do not intersect. In case (b) suppose first that not every point of $\pi_{1}$ is on $L_{1}$ or $L_{2}$. Let such a point be $P_{1}$ and adjoin as $P_{2}$ the intersection of $L_{1}$ and $L_{2}$. Here $P_{1}$ and $P_{2}$ are two points of a free extension of $\pi_{1}$ not joined by a line. Second if every point of $\pi_{1}$ is on $L_{1}$ and $L_{2}$ and there are no further lines there must be at least two points each on $L_{1}$ and $L_{2}$ since $\pi_{1}$ is nondegenerate. If these are not all joined in pairs by lines take $P_{1}$ and $P_{2}$ as a pair not joined. If they are joined in pairs adjoin a point $P_{1}$ as intersection of two of these lines not intersecting on $L_{1}$ and $L_{2}$. Third suppose here $P_{1}$ and $P_{2}$ are the points of a free extension $\pi_{1}$ and case (b) is reduced to case (a).

Now take $P_{1}$ and $P_{2}$, two points of $\pi_{1}$ (or a free extension of $\pi_{1}$ ) not joined by a line. Suppose that $\pi_{1}$ contains another pair of points $P_{3}$ and $P_{4}$ which are not joined. Then extending $\pi_{1}$, if necessary, we have:

$$
L_{2}: P_{1} P_{3} B_{2}, \quad L_{3}: P_{1} P_{4} B_{3}, \quad L_{4}: P_{2} P_{3} B_{3}, \quad L_{5}: P_{2} P_{4} B_{2}, \quad M_{3}: B_{2} B_{3} .
$$

This partial plane is necessarily complete in $\pi_{1}$ (or $\pi_{1}$ extended) since neither 
$P_{1}$ and $P_{2}$ nor $P_{3}$ and $P_{4}$ are joined. Hence by Theorem 4.3 the free extension of $\pi_{1}$ contains the free extension of (4.1). But (4.1) is a free extension of the four points $P_{1}, P_{2}, P_{3}, P_{4}$ and hence by Theorem 4.2 the free extension of $\pi_{1}$ contains a $\pi^{4}$.

In any case $\pi_{1}$, being nondegenerate, or a simple extension not involving joining $P_{1}$ and $P_{2}$, must contain two points $A_{1}$ and $A_{2}$ which together with $P_{1}$ and $P_{2}$ form a quadrilateral. We may now suppose without loss of generality that $\pi_{1}$ is generated by $P_{1}, P_{2}, A_{1}, A_{2}$ and contains

$$
\begin{aligned}
& H_{1}: P_{1} A_{1} B_{2}, \quad H_{3}: P_{2} A_{1} B_{3}, \quad H_{5}: A_{1} A_{2} \text {, } \\
& H_{2}: \quad P_{1} A_{2} B_{3}, \quad H_{4}: \quad P_{2} A_{2} B_{2}, \quad H_{6}: \quad B_{2} B_{3} \text {. }
\end{aligned}
$$

Here the points and lines are distinct but (4.2) need not be complete in $\pi_{1}$ since $H_{5}$ and $H_{6}$ may intersect in $\pi_{1}$. Now adjoin $H_{0}: P_{1} P_{2}$ and the intersections of $H_{0}$ with $H_{5}$ and $H_{6}$ to $\pi_{1}$, giving $H_{0}: P_{1} P_{2} C_{1} C_{2} H_{2}: A_{1} A_{2} C_{1} H_{6}: B_{2} B_{3} C_{2}$. Now $C_{1}$ does not join $B_{2}$ nor does $C_{2}$ join $A_{1}$. Hence $C_{1}, B_{2}$, and $C_{2}, A_{1}$ may play the role of $P_{1}, P_{2}$ and $P_{3}, P_{4}$ in (4.1) and so the free extension of $\pi_{1}$ contains a $\pi^{4}$.

TheOREM 4.5. $A \pi^{n}, n \geqq 4$, contains $a \pi^{n+1}$.

Proof. Let $\pi^{n}$ be generated by

$$
L_{1}: A_{1} A_{2}, \quad L_{6}: A_{3} A_{4}, \cdots, A_{n} .
$$

A partial free extension of (4.3) is

$$
\begin{aligned}
& L_{1}: A_{1} A_{2} B_{1} C_{5}, \quad L_{4}: A_{2} A_{3} B_{3} C_{2}, \quad M_{1}: \quad B_{1} B_{2} C_{1} C_{2} \text {, } \\
& L_{2}: A_{1} A_{3} B_{2} C_{3}, \quad L_{5}: A_{2} A_{4} B_{2} C_{4}, \quad M_{2}: B_{1} B_{3} C_{3} C_{4} \text {, } \\
& L_{3}: A_{1} A_{4} B_{3} C_{1}, \quad L_{6}: A_{3} A_{4} B_{1} C_{6} A_{5}, \cdots, A_{n}, \quad M_{3}: \quad B_{2} B_{3} C_{5} C_{6} \text {, } \\
& N_{1}: C_{1} C_{3} \text {. }
\end{aligned}
$$

This contains the partial plane complete in (4.4),

$$
L_{2}: A_{1} A_{3} C_{3}, \quad L_{3}: A_{1} A_{4} C_{1}, \quad L_{6}: A_{3} A_{4} C_{6} A_{5}, \cdots, A_{n}, \quad N_{1}: C_{1} C_{3},
$$

which is a free extension of

$$
N_{1}: \quad C_{1} C_{3}, \quad L_{6}: A_{3} A_{4} C_{6} A_{5}, \cdots, A_{n},
$$

and so $\pi^{n}$ contains the free extension of (4.5) and hence of (4.6) which is a $\pi^{n+1}$.

Now in (4.4) the triangles $A_{2} A_{3} A_{4}$ and $B_{1} B_{2} B_{3}$ are perspective with respect to $A_{1}$ and the intersections of corresponding sides are $C_{2}, C_{4}$, and $C_{6}$. But in the free extension of (4.4) these three points are not collinear. Hence a $\pi^{n}, n \geqq 4$, is never Desarguesian and in consequence of Theorem 4.4 we have the following theorem. 
Theorem 4.6. A free extension of a partial plane is never Desarguesian, provided the partial plane is nondegenerate and not a complete plane.

If from a partial plane $\pi_{2}$ we remove a point which is on exactly two lines of $\pi_{2}$ or a line which contains exactly two points we obtain a partial plane $\pi_{1}$ of which $\pi_{2}$ is a free extension. This removal, or any succession of such removals, will be called a free contraction. If one partial plane may be obtained from another by any succession of free extensions or free contractions we say that the two partial planes are free-equivalent. It is easily shown that this is a true equivalence, being reflexive, symmetric, and transitive.

THEOREM 4.7. A free contraction followed by a free extension may be replaced by a free extension followed by a free contraction.

Proof. It is sufficient to prove this when there is only one step in the contraction and one in the extension. If we remove a point $P$ and adjoin a line $L$ we may first adjoin $L$ and then remove $P$, since $P$ was not used to define $L$, and the two lines which intersected in $P$ have not been altered, nor does $L$ pass through $P$. If we remove a point $P$ and adjoin a point $Q \neq P$, we may first adjoin $Q$ and then remove $P$, since the two lines for which $Q$ is an intersection do not intersect before the removal of $P$. If we remove $P$ and then adjoin $Q=P$, we may simply omit both steps or adjoin any element and then remove it. The situation is similar if the element first removed is a line.

A finite partial plane which has three or more points on every line and three or more lines through every point is said to be confined. Free contraction is not possible in a confined partial plane, or confined configuration as we may call it.

Theorem 4.8. A free plane does not contain any confined configuration.

Proof. The generating partial plane (4.3) of a free plane $\pi^{n}$ does not contain any confined configuration since there are only two lines. Hence a confined configuration $\alpha$ must contain elements of later stages. Consider an element of the last stage occurring in $\alpha$. Suppose it to be a line $L$ of the $i$ th stage. The points on it must be of earlier stages. But in a free plane an $i$ th stage line contains only two points of earlier stages, namely those defining it, and we have a contradiction. Similarly if an element of the last stage in $\alpha$ is a point we have a contradiction. Hence $\pi$ contains no confined configuration.

We shall call a finite partial plane which contains no confined configuration an open configuration. Here is an example of an open configuration in which free contraction is not possible, but which becomes quite simple under free equivalence.

$$
\begin{aligned}
& L_{1}: A_{1} A_{2} C_{1}, \quad L_{3}: A_{1} A_{4} B_{3}, \quad L_{5}: A_{2} A_{4} B_{2}, \\
& L_{2}: A_{1} A_{3} B_{2}, \quad L_{4}: A_{2} A_{3} B_{3}, \quad L_{6}: A_{3} A_{4} C_{2} \text {, } \\
& L_{7}: \quad B_{2} B_{3} C_{3} \text {. }
\end{aligned}
$$


By free extension

$$
\begin{array}{cllll}
L_{1}: & A_{1} A_{2} C_{1}, & L_{4}: & A_{2} A_{3} B_{3} D_{1} D_{2} D_{3}, & L_{7}: B_{2} B_{3} C_{3}, \\
L_{2}: & A_{1} A_{3} B_{2}, & L_{5}: A_{2} A_{4} B_{2}, & M_{1}: A_{4} C_{1} D_{1}, \\
L_{3}: & A_{1} A_{4} B_{3}, & L_{6}: A_{3} A_{4} C_{2}, & M_{2}: A_{1} C_{2} D_{2}, \\
& & M_{3}: A_{1} C_{3} D_{3} .
\end{array}
$$

By free contraction we may remove first $C_{1}, C_{2}, C_{3}$ then $M_{1}, M_{2}, M_{3}, L_{1}, L_{6}, L_{7}$ and then $B_{2}, B_{3}$ and $L_{2}$ and $L_{5}$ leaving

$$
L_{3}: A_{1} A_{4}, \quad L_{4}: A_{2} A_{3} D_{1} D_{2} D_{3},
$$

the open configuration generating $\pi^{7}$. The general problem of free equivalence is treated in the following theorem.

THEOREM 4.9. A nondegenerate open configuration is free equivalent to a configuration (4.3) which generates $a \pi^{n}$.

Proof. Given an open configuration which is nondegenerate. Extending, if necessary, we may find a quadrilateral $A_{1}, A_{2}, A_{3}, A_{4}$ and hence by free equivalence we will have a configuration:

$$
\begin{array}{ll}
L_{1}: & A_{1} A_{2}\left(C_{1}\right) \cdots, \quad\left[B_{1}, \cdots, B_{s}\right], \\
L_{2}: & A_{3} A_{4}\left(C_{1}\right) \cdots, \\
L_{3}: & , \cdots, L_{r}:,
\end{array}
$$

where the intersection $C_{1}$ of $L_{1}$ and $L_{2}$ may or may not occur, and the points $B_{1}, \cdots, B_{s}$ are on no line. We shall reduce the configuration in various ways by free equivalence, by reducing the number of lines, or keeping the number of lines fixed, always retaining two lines such as $L_{1}$ and $L_{2}$ containing a quadrilateral. This reduction process must come to an end in a finite number of steps and we shall show that the end is not reached as long as there are three or more lines, and then treat the cases with two lines.

First if there is a line $L$ with no points, intersect $L$ with $L_{1}$ and $L_{2}$ and then remove $L$. If there is a line $L$ with exactly one point not equal to $A_{1}, A_{2}, C_{1}$, intersect $L$ with $L_{1}$ and then remove $L$. If there is a line $L$ with exactly one point, either $A_{1}$ or $A_{2}$, intersect $L$ with $L_{2}$ and then remove $L$. If there is a line $L$ with exactly the one point $C_{1}$ adjoin $M: A_{1} A_{3}$ if necessary, intersect with $L$ and then remove $L$. Naturally we remove any line $L_{3}, \cdots, L_{r}$ with exactly two points. Hence we may suppose that either a reduction is possible or that each of the lines $L_{3}, \cdots, L_{r}$ contains at least three points. Now if some line, say $L_{3}$, contains a point $A_{5} \neq A_{1}, A_{2}, A_{3}, A_{4}$ which occurs exactly once, then $L_{3}$ cannot contain both $A_{1}$ and $A_{2}$, and say it does not contain $A_{1}$. Then $M: A_{1} A_{5}$ will be a new line. By free extension (4.10) becomes 


$$
\begin{aligned}
& L_{1}: A_{1} A_{2}\left(C_{1}\right) \cdots \quad\left[B_{1}, \cdots, B_{s}\right], \\
& L_{2}: A_{3} A_{4}\left(C_{1}\right) \cdots D_{1}, \\
& L_{3}: A_{5} A_{6} \cdots, \cdots, L_{r}: \quad, \quad M: A_{1} A_{5} D_{1} .
\end{aligned}
$$

By free contraction it becomes

$$
\begin{array}{ll}
L_{1}: & A_{1} A_{2}\left(C_{1}\right) \cdots \quad\left\lfloor B_{1}, \cdots, B_{s}\right\rfloor, \\
L_{2}: & A_{3} A_{4}\left(C_{1}\right) \cdots D_{1}, \\
L_{3}: & A_{6} \cdots, \cdots, L_{r}: .
\end{array}
$$

Hence a point $A_{5}$ occurring exactly once on $L_{3}$ may be replaced by a point occurring exactly once on $L_{2}$ (or similarly on $L_{1}$ ). Since this process is clearly reversible, we may shift the points of single occurrence at will and we shall suppose that they are all on one line, either $L_{1}$ or $L_{2}$ according to preference. Note that this does not involve any increase in the number of points. We remove any point occurring exactly twice, except one of $A_{1}, A_{2}, A_{3}, A_{4}$. If one of these occurs exactly twice, suppose this to be $A_{1}$ occurring a second time on $L_{3}$ and that the points of single occurrence are on $L_{2}$. Now $L_{3}$ cannot contain $C_{1}$ and if it contains neither $A_{3}$ nor $A_{4}$, then $L_{3}: A_{1} A_{5} A_{6} \cdots$ where $A_{3}, A_{4}, A_{5}, A_{6}$ form a quadrilateral and we may reduce by dropping $A_{1}$ and keeping $L_{2}, L_{3}$ in the role of $L_{1}, L_{2}$. If $L_{3}$ contains one of $A_{3}, A_{4}$ say $A_{3}$, then $L_{3}: A_{1} A_{3} A_{5} \cdots$ and this reduction fails. Here if $C_{1}$ occurs, then in its third occurrence $L_{4}: C_{1} A_{6} A_{7} \cdots$ and we may take $A_{3}, A_{4}, A_{6}, A_{7}$ as a quadrilateral on $L_{2}$ and $L_{4}$ and reduce by dropping $A_{1}$. If $C_{1}$ does not occur, then $A_{5}$ occurs on at least two lines besides $L_{3}$ and at least one of these is not through $A_{4}$, say $L_{5}: A_{5} A_{6} \ldots$. Here we may take $A_{3}, A_{4}, A_{5}, A_{6}$ as a quadrilateral and drop $A_{1}$ for a reduction. Hence if there are more than two lines and (4.10) is not reducible by one of these devices, we may suppose that every point occurs either once or at least three times, that the points of single occurrence are on $L_{1}$ or $L_{2}$, and that each of $L_{3}, \cdots, L_{r}$ contains at least three points. Since there are at least three points on $L_{3}$ and since each of these occurs at least three times, there must be a fourth line to yield enough incidences. Now since $L_{3}$ and $L_{4}$ have three or more points each, points occurring three or more times must contain a quadrilateral of points each occurring three or more times. Hence shifting from $A_{1}, A_{2}, A_{3}, A_{4}$ to this quadrilateral, if necessary, we may assume that $A_{1}, A_{2}, A_{3}, A_{4}$ each occurs at least three times in (4.10) or that further reduction is possible. Now if in (4.10) we suppress all points of single occurrence, the remaining configuration must, by hypothesis, be open. But this leaves $L_{3}, \cdots, L_{r}$ with at least three points each, $L_{1}$ with at least $A_{1}, A_{2}$ and $L_{2}$ with at least $A_{3}, A_{4}$ and every point occurring three or more times. Hence one of $L_{1}, L_{2}$, say $L_{1}$, can have no more than the two points $A_{1}, A_{2}$. Now in (4.10), without suppression of points of single occurrence, the single points may be supposed shifted to $L_{2}$. Since there are no 
points of double occurrence, this leaves $L_{1}$ with exactly the two points $A_{1}, A_{2}$. $L_{3}$ and $L_{4}$ will certainly contain a quadrilateral and so we may reduce by dropping $L_{1}$ and still keep two lines with a quadrilateral. This completes reduction of (4.10) by free equivalence to a configuration with two lines containing a quadrilateral,

$$
L_{1}: A_{1} A_{2} D_{1} \cdots D_{t}\left[B_{1}, \cdots, B_{s}\right], \quad L_{2}: A_{3} A_{4} \cdots A_{u},
$$

where $C_{1}$ has been removed if it occurred. By free extension

$$
\begin{array}{lll}
L_{1}: & A_{1} A_{2} D_{1} \cdots D_{t}\left[B_{2}, \cdots, B_{8}\right], & L_{3}: A_{1} B_{1} A_{u+1}, \\
L_{2}: & A_{3} A_{4} \cdots A_{u} A_{u+1} A_{u+2}, & L_{4}: A_{2} B_{1} A_{u+2} .
\end{array}
$$

By free contraction we remove $B_{1}$ and then $L_{3}, L_{4}$, yielding

$$
\dot{L_{1}}: A_{1} A_{2} D_{1} \cdots D_{t}\left[B_{2}, \cdots, B_{8}\right], \quad L_{2}: \quad A_{3} A_{4} \cdots A_{u+2} .
$$

Thus each $B$ may be replaced by two $A$ 's on $L_{2}$ until we have

$$
L_{1}: A_{1} A_{2} D_{1} \cdots D_{t}, \quad L_{2}: A_{3} A_{4} \cdots A_{v} .
$$

Now we may use a free extension

$$
\begin{array}{lll}
L_{1}: & A_{1} A_{2} D_{1} \cdots D_{t}, & L_{3}: A_{1} A_{3} E_{1}, \\
L_{2}: & A_{3} A_{4} \cdots A_{v} A_{v+1}, & L_{4}: A_{2} A_{4} E_{1},
\end{array}
$$

$$
L_{5}: D_{t} E_{1} A_{v+1} \text {, }
$$

where by free contraction we may remove $D_{t}$, then $L_{5}$, then $E_{1}$, and finally $L_{3}$ and $L_{4}$ leaving

$$
L_{1}: A_{1} A_{2} D_{1} \cdots D_{t-1}, \quad L_{2}: A_{3} A_{4} \cdots A_{v+1} .
$$

In this way we may replace every $D$ by another $A$ on $L_{2}$ reaching

$$
L_{1}: A_{1} A_{2}, \quad L_{2}: A_{3} A_{4} \cdots A_{n},
$$

which we may call the free $n$-point. This completes the proof of the theorem.

In any configuration let $P$ be the number of points, $L$ the number of lines, and $I$ the number of incidences.

THEOREM 4.10. The number $2(P+L)-I$ is invariant under free equivalence.

Proof. In free extension, adding a point we increase $P$ by 1 and $I$ by 2 . Adding a line, we increase $L$ by 1 and $I$ by 2 . In free contraction we decrease by similar amounts. In all cases $2(P+L)-I$ remains unchanged.

THEOREM 4.11. An open configuration $\alpha$ which is nondegenerate is free equivalent to the free $n$-point if and only if

$$
2(P+L)-I=n+4 .
$$


Proof. By Theorem 4.9, $\alpha$ is free-equivalent to some free $n$-point and by Theorem 4.10, $2(P+L)-I$ is invariant under free equivalence. Now for (4.19) $P=n, L=2, I=n$ and $2(P+L)-I=n+4$.

THEOREM 4.12. $A$ free $n$-point and a free $m$-point are not free equivalent if $m \neq n$. The planes $\pi^{m}$ and $\pi^{n}$ are not isomorphic.

An immediate consequence of Theorems 4.10 and 4.11 is that the free $m$-point and the free $n$-point are not free equivalent if $m \neq n$. If $\pi^{m}$ and $\pi^{n}$ were isomorphic we could consider both as the same plane generated in one way by a free $m$-point and in another by a free $n$-point. Let $C_{1}$ be a configuration generated by the free $m$-point large enough to include the generating free $n$-point. Then extend $C_{1}$ by generating from the free $n$-point so that the generating free $m$-point is constructible from the free $n$-point. Call the extended configuration $C_{2}$. Now from its method of construction $C_{2}$ may be considered as generated either by the free $m$-point or by the free $n$-point. By assumption the whole plane is the free extension of both the $m$-point and the $n$-point. Hence by free contraction $C_{2}$ is free-equivalent to both the $m$-point and the $n$-point, and they are free-equivalent to each other. But this is a contradiction and hence $\pi^{m}$ and $\pi^{n}$ cannot be isomorphic.

TheOREM 4.13. A plane containing no confined configuration and generated by a finite configuration is a free plane $\pi^{n}$.

CoROllaRY. The finitely generated subplanes of a free plane are free.

Proof. Let $\pi$ contain no confined configurations, and be generated by a finite configuration $\alpha$. In the extension of $\alpha$ the free adjunction of a point or line leaves $2(P+L)-I$ invariant, while the adjunction of a point on three or more lines or a line through three or more points decreases the value of $2(P+L)-I$. Now a configuration for which $2(P+L)-I \leqq 7$ cannot by Theorem 4.11 be an open configuration. Hence in any (finite) extension of $\alpha$ in $\pi$, $2(P+L)-I \geqq 8$ and consequently in generating $\pi$ from $\alpha$ there are only a finite number of adjunctions which are not free. Hence $\pi$ may be considered the free extension of some finite extension $\beta$ of $\alpha$. But $\beta$ is an open configuration and hence a finite extension $\gamma$ of $\beta$ is a free extension of a free $n$-point and hence $\pi$, as a free extension of $\gamma$, is a free plane $\pi^{n}$.

It is conceivable that Theorem 4.13 would not be true without the assumption of the finiteness of $\alpha$. It is possible that the generators of a plane might be so scattered and so interrelated that it might be impossible to find points on a single line and two points off it which would generate the entire plane without any relation between them.

It is easy to prcve by the method of Theorem 4.8 that a free extension of a partial plane contains no confined configuration except those included entirely in the partial plane. Thus any collineation or duality of the free 
extension of a confined configuration maps the confined configuration into itself. In this way it is not too difficult to construct planes which are not selfdual, planes with only the identity as a collineation, and so on. Further investigation along these lines might be rewarding.

5. Introduction of coordinates. Classical investigations $\left({ }^{6}\right)$ have shown that in a Desarguesian plane we may introduce homogeneous coordinates from a skew-field and thereafter proceed by the methods of analytic geometry. For a given skew-field $F$, we denote points by $P\left(\lambda x_{1}, \lambda x_{2}, \lambda x_{3}\right), x_{1}, x_{2}, x_{3}$ not all zero, $\lambda \neq 0$ arbitrary. We denote lines by $L\left[a_{1} \sigma, a_{2} \sigma, a_{3} \sigma\right], a_{1}, a_{2}, a_{3}$ not all zero, and $\sigma \neq 0$ arbitrary. Here $P \subset L$ if and only if

$$
x_{1} a_{1}+x_{2} a_{2}+x_{3} a_{3}=0 .
$$

Given any skew-field $F$ it is not difficult to verify that points and lines so defined form a Desarguesian plane. We shall distinguish between an analytic plane and a Desarguesian plane until the equivalence has been shown here. It is not the purpose of this paper to duplicate this classic result, but it will be incidental to other investigations.

In an analytic plane it is easy to verify that the projective transformations

$$
\begin{aligned}
& \rho x_{1}=b_{11} x_{1}^{\prime}+b_{12} x_{2}^{\prime}+b_{13} x_{3}^{\prime}, \\
& \rho x_{2}=b_{21} x_{1}^{\prime}+b_{22} x_{2}^{\prime}+b_{23} x_{3}^{\prime}, \\
& \rho x_{3}=b_{31} x_{1}^{\prime}+b_{32} x_{2}^{\prime}+b_{33} x_{3}^{\prime},
\end{aligned}
$$

with $\rho \neq 0$ and the matrix $\left(b_{i j}\right)$ of rank three, form a group of collineations and that by an appropriate collineation the vertices of any quadrilateral may be taken in to $A(0,1,0), B(1,0,0), C(0,0,1)$, and $D(1,1,1)$. In the following section we shall select an arbitrary quadrilateral in any projective plane $\pi$ and introduce coordinates with respect to which it plays a role similar to that of $A B C D$ in the analytic plane.

Let us select any two distinct points $A$ and $B$ in the projective plane $\pi$. If from $\pi$ we remove the line $L_{\infty}$ joining $A$ and $B$ and the points on $L_{\infty}$, the remaining plane $\pi^{*}$ is a Euclidean plane. We shall use $A$ and $B$ as centers of perspectivities to introduce coordinates for the points of $\pi^{*}$. No ambiguity will arise if, as we shall find convenient on occasion, we speak of $\pi$ and $\pi^{*}$ as the same plane so long as $L_{\infty}$ is fixed.

Let the lines of the pencil through $A$ in $\pi^{*}$ be denoted by $x=x_{0}, \cdots, x=x_{n-1}$ where $n$ is their cardinal number and $x_{0}, \cdots, x_{n-1}$ are $n$ different symbols. Similarly denote the lines of the pencil through $B$ by $y=y_{0}, \cdots, y=y_{n-1}$. (The cardinal number of the $y$ 's is necessarily the same as that of the $x$ 's.) Then through every point $P$ of $\pi^{*}$ there is exactly one line $x=x_{i}$ and one line $y=y_{j}$. Denote $P$ by $\left(x_{i}, y_{j}\right)$. As in Figure 3 we see that on an arbitrary line $L$ of $\pi^{*}$ not through $A$ or $B$ there are points $\left(x_{i}, y_{i}\right)$ where each $x$ and each $y$

(') D. Hilbert [1], K. G. C. von Staudt [1], vol. 2, pp. 166 et seq. 
occurs exactly once. Henceforward suppose that the symbols $x_{0}, \cdots, x_{n-1}$ are the same as $y_{0}, \cdots, y_{n-1}$, though not necessarily in the same order. Then an arbitrary line $L$ not through $A$ or $B$ is associated with the permutation $\left(\ldots, x_{i}, \cdots\right)$ where the $\left(x_{i}, y_{i}\right)$ are the points of $L$. This expresses the fact (see Figure 3) that $L$ determines a 1-1 correspondence between the lines of the pencil through $A$ and the lines of the pencil through $B$. Without confusion we may write

$$
L=\left(\ldots, x_{i}, \cdots\right)
$$

since distinct lines contain at most one point in common and hence are associated with distinct permutations.

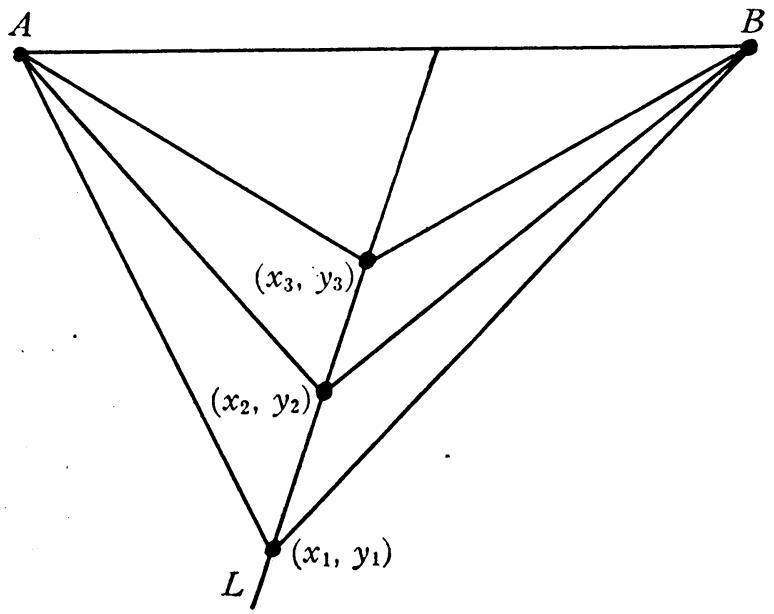

FIG. 3

THEOREM 5.1. The projection $L \rightarrow_{A} M \rightarrow_{B} L$ induces the permutation $\left(x_{i}, y_{i}\right) \rightarrow\left(x_{j}, y_{j}\right)$ of the points of $L$ where $\left(\ldots, x_{i}, \cdots\right)=M L^{-1}$.

Proof. The perspectivity $L \rightarrow_{A} M$ takes $\left(x_{i}, y_{i}\right)$ of $L$ into $\left(x_{i}, z_{i}\right)$ of $M$ and the perspectivity $M \rightarrow{ }_{B} L$ takes $\left(x_{i}, z_{i}\right)$ of $M$ into $\left(x_{j}, y_{j}\right)$ of $L$ where $y_{j}=z_{i}$. But $M=\left(\cdots, x_{i}, \cdots\right)$ and $L^{-1}=\left(\cdots, \cdots, y_{j}, \cdots\right)$ where $z_{i}=y_{j}$ and hence $M L^{-1}$ $=\left(\ldots, x_{i}, \cdots\right)$.

We have shown that in an arbitrary Euclidean plane $\pi^{*}$ the points may be represented by $\left(x_{i}, y_{j}\right)$ and the lines by $x=c_{i}, y=c_{j}$ and permutations $L$ of $n$ symbols $c_{0}, \cdots, c_{n-1}$. What are the properties that characterize the set $S$ of permutations $[L]$ ? This question is answered by the following theorem.

TheOREM 5.2. A set $S$ of permutations on $n$ letters $c_{0}, \cdots, c_{n-1}$ will be associated with the lines of a Euclidean plane $\pi^{*}$ not through its centers of perspectivity if and only if (a), (b), and (c) or ( $\left.\mathrm{c}^{\prime}\right)$ hold. 
(a) $S$ is doubly transitive on $c_{0}, \cdots, c_{n-1}$.

(b) If $M$ and $L$ are different permutations of $S$, then $M L^{-1}$ fixes at most one letter.

(c) If $L$ does not take $c_{i}$ into $c_{j}$ there is one and only one permutation $M$ which does take $c_{i}$ into $c_{j}$ such that $M L^{-1}$ displaces all letters.

(c') $n$ is finite.

REMark. Note that an interchange of the centers of perspectivity, $A$ and $B$, replaces $S$ by $S^{-1}$, the set of inverses of elements in $S$, and that $S^{-1}$ satisfies conditions (a), (b), and (c) if and only if $S$ satisfies them.

Proof. In this proof we shall use conditions (a), (b), and (c). See appendix I for a proof that (a), (b), and ( $\left.c^{\prime}\right)$ imply (c) and also for counter-examples showing that when $n$ is infinite (a) and (b) do not imply (c).

Suppose $S$ is the set of permutations associated with lines $L$ not through $A$ or $B$ in a Euclidean plane $\pi^{*}$. If $x_{1}, x_{2}$ are two different symbols and $y_{1}, y_{2}$ are two different symbols then there is a line $L$ through $\left(x_{1}, y_{1}\right)$ and $\left(x_{2}, y_{2}\right)$, and since $x_{1} \neq x_{2}$ and $y_{1} \neq y_{2}$ this line does not pass through $A$ or $B$. Hence $L=\left(\begin{array}{l}x_{1}, x_{2}, \cdots \\ v_{1}, y_{2}, \cdots\end{array}\right)$ and (a) is satisfied. Now if $M$ and $L$ are two different lines $M L^{-1}$ fixes a letter $c_{i}$ if and only if $M$ and $L$ both take $c_{i}$ into the same letter, say $c_{j}$, whence $M$ and $L$ have the point $\left(c_{i}, c_{j}\right)$ in common. Since $M$ and $L$ have at most one point in common $M L^{-1}$ can fix at most one letter. Hence (b) is satisfied. Now if $L$ does not take $c_{i}$ into $c_{j}$, that is if $\left(c_{i}, c_{j}\right)$ is not on $L$, then there is exactly one line $M$ parallel to $L$ through $\left(c_{i}, c_{j}\right)$, whence $M$ takes $c_{i}$ into $c_{j}$ and $M L^{-1}$ displaces all letters. Hence (c) is satisfied.

Conversely suppose $S$ satisfies conditions (a), (b), and (c). Construct points $\left(x_{i}, y_{j}\right)$ and lines $x=c_{i}, y=c_{j}$ and $L=\left(\cdots, x_{i}, \ldots\right)$ with $L$ from $S$. Then the points and lines of this system satisfy the axioms for a Euclidean plane $\pi^{*}$. Using (a) we may verify that there is a line through two arbitrary points. From (b) we may show that two lines intersect in at most one point. From (c) it follows that through a given external point there is exactly one line parallel to a given line. In the verification of these axioms, the lines $x=c_{i}$ and $y=c_{j}$ form two sets of parallels and assure us that there is a line through $\left(x_{1}, y_{1}\right)$ and $\left(x_{2}, y_{2}\right)$ even if $x_{1}=x_{2}$ or $y_{1}=y_{2}$.

In a plane any two lines contain the same number of points (here $n$ for the Euclidean plane, $n+1$ for the projective plane). Hence if two planes are isomorphic the number of points per line must be the same for both. Since in Theorem 5.2 the symbols $c_{0}, \cdots, c_{n-1}$ are any $n$ distinct symbols, without loss of generality we may assume that the same symbols are used in representing any two planes which have the same number of points on a line.

Theorem 5.3. The plane $\pi_{1}$ represented on the pencils through $A_{1}$ and $B_{1}$, using symbols $c_{0}, \cdots, c_{n-1}$, will be isomorphic to the plane $\pi_{2}$ represented on the pencils through $A_{2}$ and $B_{2}$, using symbols $c_{0}, \cdots, c_{n-1}$, with $A_{1} \rightleftarrows A_{2}$ and $B_{1} \rightleftarrows B_{2}$, if and only if there exist two permutations $X$ and $Y$ of $c_{0}, \cdots, c_{n-1}$ such that 
$X^{-1} S_{1} Y=S_{2}$, where $S_{1}$ is the set of $L$ permutations of $\pi_{1}$ and $S_{2}$ is the set of $L$ permutations of $\pi_{2}$. Here $X=\left(\cdots, \ldots, c_{i}, \cdots\right)$ where the line $x=c_{i}$ of the $A_{1}$ pencil is mapped onto the line $x=c_{j}$ of the $A_{2}$ pencil, and $Y=\left(\cdots, c_{r}, \cdots\right)$ where the line $y=c_{r}$ of the $B_{1}$ pencil is mapped onto the line $y=c_{s}$ of the $B_{2}$ pencil.

Proof. Suppose there is an isomorphism $\pi_{1} \rightleftarrows \pi_{2}, A_{1} \rightleftarrows A_{2}, B_{1} \rightleftarrows B_{2}$. Then the pencil through $A_{1}$ is mapped onto the pencil through $A_{2}$, and that through $B_{1}$ mapped onto that through $B_{2}$. Hence correspondences $x=c_{i} \rightleftarrows x=c_{j}$ and $y=c_{r} \rightleftarrows y=c_{s}$ are determined. Write $X=\left(\cdots, c_{i}, \cdots\right), \quad Y=\left(\ldots, c_{r}, \cdots\right)$. Then $P_{1}\left(c_{i}, c_{r}\right) \rightleftarrows P_{2}\left(c_{j}, c_{s}\right)$. Here if $P_{1} \subset L_{1}$ and $L_{1} \rightleftarrows L_{2}$, then $P_{2} \subset L_{2}$. Hence if the permutation $L_{1}$ takes $c_{i}$ into $c_{r}$ then $X^{-1} L_{1} Y$ takes $c_{j}$ into $c_{s}$ whence $L_{2}=X^{-1} L_{1} Y$ as was to be shown. Conversely suppose that $X^{-1} S_{1} Y=S_{2}$. Then let us set up the correspondences $A_{1} \rightleftarrows A_{2}, B_{1} \rightleftarrows B_{2}, x=c_{i} \rightleftarrows x=c_{j}, y=c_{r} \rightleftarrows y=c_{s}$ and $P_{1}\left(c_{i}, c_{r}\right)$ $\rightleftarrows P_{2}\left(c_{j}, c_{s}\right)$. Also put $L_{1} \rightleftarrows L_{2}$ if $L_{2}=X^{-1} L_{1} Y$. Here $P_{1} \subset L_{1}$ if and only if $P_{2} \subset L_{2}$. Hence this is an isomorphism between $\pi_{1}$ and $\pi_{2}$, which maps $A_{1}$ onto $A_{2}$ and $B_{1}$ onto $B_{2}$.

We may use the permutations $S$ associated with a plane $\pi$ to introduce as another representation of $\pi$ a ternary ring. This ternary ring, as will be proved later, reduces to the usual coordinate ring in an analytic plane, but, without conditions of Desarguesian type, it will have very few of the ordinary ring properties of a skew-field. Among the various equivalent representations of $\pi$ which Theorems 5.2 and 5.3 yield we may, by taking $X=L, Y=I$, obtain a representation in which an arbitrary line $L \rightleftarrows L^{-1} L I=I$ is represented by the identity. Now take two arbitrary symbols as $c_{0}=0$, and $c_{1}=1$. We keep unchanged the representation of the lines $x=c_{i}, y=c_{j}$. The remaining lines $M$ we use to define a ternary operation $y=x \cdot m \circ b$ on the symbols $0,1, c_{2}, \cdots, c_{n-1}$. To a given line $M$ correspond parameters $m, b$ in the following way:

(1) If $M=\left(\begin{array}{l}0,1, \cdots) \\ 0, s, \cdots\end{array}\right)$, then for $M$ take $m=s, b=0$.

(2) If $M$ goes through $(0, a), a \neq 0$, and is parallel to $\left(\begin{array}{c}0,1, \cdots \\ 0, s, \ldots)\end{array}\right)$ then for $M$ take $m=s, b=a$. If $(x, y)$ is any point on $M$ define the ternary operation as $y=x \cdot m \circ b$. Here a point is represented by a pair of coordinates $(x, y)$ and a line contains all those points bound by the ternary relation $y=x \cdot m \circ b$. This operation is defined for all $x, m, b$ selected from the system $0,1, c_{2}, \cdots, c_{n-1}$ except for triples with $m=0$. We may extend slightly by defining $x \cdot 0 \circ b=b$ for any $x$ and thus include the lines $y=c_{j}$ in the general form. In the analytic planes $(0, b)$ is the intercept of $M$ on the $y$-axis, $m$ is the slope of $M$, and the equation of $M$ is $y=x m+b$. What in general are the properties of this ternary operation and when does such a ternary operation define a plane?

THEOREM 5.4( $\left.{ }^{7}\right)$. A ternary operation $a \cdot b \circ c$ uniquely defined for all ordered triples $a, b, c$ containing elements 0 and 1 may be derived from the incidences of $a$

(7) Similar results, as yet unpublished, have been obtained by D. T. Perkins. 
Euclidean plane having the following properties:

(1) $0 \cdot m \circ c=a \cdot 0 \circ c=c, 1 \cdot m \circ 0=m, a \cdot 1 \circ 0=a$.

(2) $x \cdot m \circ b=c$, for given $m \neq 0, b, c$, has a unique solution $x$.

(3) $a \cdot m \circ z=c$, for given $a, m, c$, has a unique solution $z$.

(4) $a \cdot z \circ b=c$, for given $a \neq 0, b, c$, has a unique solution $z$.

(5) $x \cdot m_{1} \circ b_{1}=x \cdot m_{2} \circ b_{2}$ has a unique solution $x$ if $m_{1} \neq m_{2}$.

(6) The pair of equations $x_{1} \cdot m \circ b=y_{1}, x_{2} \cdot m \circ b=y_{2}$ has a unique solution $m=s, b=a$ given $x_{1} \neq x_{2}, y_{1} \neq y_{2}$.

For each choice of a fundamental quadrilateral $A, B, O=(0,0), I=(1,1)$ in $\pi$ the ternary operation is uniquely determined. Conversely a ternary operation on $a$ system $T$ with the above properties defines a Euclidean plane with points $(a, b)$, $a, b \subset T$, and lines $x=c$ and $y=x \cdot m \circ b$.

Proof. As was shown above, a plane may be represented as in Theorem 5.2, taking one line $L$ as the identity, the coordinates being from a system $T\left(0,1, c_{2}, \cdots, c_{r-1}\right)$. The points are $\left(c_{i}, c_{j}\right)$ and the lines are $x=c_{i}, y=x \cdot m \circ b$. Here $a \cdot 0 \circ c=c$ is part of the definition of the ternary operation and $0 \cdot m \circ c=c$ states that $(0, c)$ is on $y=x \cdot m \circ c .1 \cdot m \circ 0=m$ states that $(1, m)$ is on $\left(\begin{array}{l}0,1, \ldots) \\ 0, m, \ldots\end{array}\right)$ if $m \neq 0$ while $1 \cdot 0 \circ 0=0$ is a special case of $a \cdot 0 \circ c=c$. $a \cdot 1 \circ 0=a$ states that $\left(\begin{array}{l}0,1, \cdots \\ 0,1, \cdots\end{array}\right)$ is the identical permutation. So much for property (1). (2) states that $y=c$ intersects $\left(\begin{array}{l}0, \cdots \\ b, \cdots\end{array}\right)$ in exactly one point. In (3) if $m=0, a \cdot m \circ z=z=c$ and $z$ is unique. If $m \neq 0$ it states that there is a unique parallel to $\left(\begin{array}{l}0,1, \cdots \\ 0, m, \cdots\end{array}\right)$ through $(a, c)$. (4) states that if $a \neq 0$ and $b=c$ then $z=0$ is a solution, whereas if $a \cdot m \circ b=c$ with $m \neq 0$ we have $\left(\begin{array}{l}0, a, \cdots \\ b, c, \ldots\end{array}\right)$ parallel to $\left(\begin{array}{c}0,1, \cdots \\ 0, m, \cdots\end{array}\right)$, hence $c \neq b$. If $b \neq c$ there is a unique parallel $\left(\begin{array}{l}0,1, \ldots \\ 0, m\end{array}, \ldots\right)$ through $(0,0)$ to $\left(\begin{array}{l}0, a, \cdots \\ b, c, \cdots\end{array}\right)$, and $z=m$ is the unique solution. (5) states that the lines $y=x \cdot m_{1} \circ b_{1}$ and $y=x \cdot m_{2} \circ b_{2}$, if $m_{1} \neq m_{2}$, are not parallel and intersect in a unique point $(x, y)$. (6) states that there is a unique line through $\left(x_{1}, y_{1}\right)$ and $\left(x_{2}, y_{2}\right)$ if $x_{1} \neq x_{2}, y_{1} \neq y_{2}$. Hence the ternary operation defined by a plane has properties (1) to (6) inclusive.

Conversely suppose that in a system $T\left(0,1, c_{2}, \cdots, c_{n-1}\right)$ a ternary operation $a \cdot b \circ c$ is defined satisfying properties (1) to (6). Let us define points $\left(c_{i}, c_{j}\right)$ and lines $x=c_{i}, y=c_{j}=x \cdot 0 \circ c_{j}$, and lines $M(m, b)=\left(\ldots, \ldots, c_{i}, \cdots\right)$ if $c_{j}=c_{i} \cdot m \circ b$ for every $m \neq 0$, and every $b$. Since $x \cdot m \circ b$ is uniquely defined for every $x$ in $M, c_{i}$ takes on every value exactly once. From property (2) every $c_{j}$ occurs exactly once in $M$ and so $M$ is a permutation of the $c$ 's. From property (6) the permutations $M$ are doubly transitive. From property (5) $M_{1} M_{2}^{-1}$ fixes exactly one letter if the slopes $m_{1}$ and $m_{2}$ are different. If $m_{1}=m_{2}=m, b_{1} \neq b_{2}$, $x \cdot m \circ b_{1} \neq x \cdot m \circ b_{2}$ since from property (3) $a \cdot m \circ z=c$ has a unique solution, and in this case $M_{1} M_{2}^{-1}$ fixes no letter. If $m_{1}=m_{2}$ and $b_{1}=b_{2}$, the lines $M_{1}$ and $M_{2}$ are identical. From the definition of the ternary operation and property (2) it follows that no line $y=x \cdot m \circ b$ with $m \neq 0$ can be parallel to a line $x=c$ or $y=c$. Hence through an external point there is exactly one parallel to a 
line of slope $m$ whose slope is $m$ (property 3 ), and no parallel with a different slope. Hence the conditions (a), (b), (c) of Theorem 5.2 are all satisfied and the ternary relation does in fact define a plane. In addition, one of the lines, $y=x \cdot 1 \circ 0$, is the identity $y=x$. Note that in this coordinatization of the plane not only $A$ and $B$, but also $(0,0)$ and $(1,1)$ play a special role and determine from the plane the ternary operation to within isomorphism. Different choices of the fundamental quadrilateral may lead to systems not isomorphic in any algebraic sense. For examples, see appendix II.

We may define both addition and multiplication as special cases of the ternary operation.

Addition: $a+b=c$ if and only if $a \cdot 1 \circ b=c$.

Multiplication: $a b=c$ if and only if $a \cdot b \circ 0=c$. Here we have $0+a=a+0=a$ and the existence of unique $u, v$ such that $u+a=b, a+v=b$, though not even the right and left negatives of $a$ need be equal. Also $a 0=0 a=0$ and $a 1=1 a=a$, while for $a \neq 0, u a=b, a v=b$ have unique solution's, though not even the right and left inverses need be the same. In general no other ring properties hold, nor will it be true that $x \cdot m \circ b=x m+b$.

In an affine analytic plane lines $y=x m+b$ with $m \neq 0$ may be associated with the transformations $x \rightarrow x m+b$ of the linear group. Here the permutations $(\cdots, \cdots, \cdots)$ will be the elements of the linear group. Hence when skewfield coordinates have been introduced, the permutations of Theorem 5.2 form a group. But it is possible for these permutations to form a group even in certain non-analytic planes. See appendix II for an example of such a plane.

THEOREM 5.5. If the $A_{1}-B_{1}$ permutations of a plane $\pi_{1}$ form a group $G$, the $A_{2}-B_{2}$ permutations of a flane $\pi_{2}$ isomorphic to $\pi_{1}$ with $A_{2} \rightleftarrows A_{1}, B_{2} \rightleftarrows B_{1}$ form a coset of a transform of $G$ in the symmetric group on $c_{0}, \cdots, c_{n-1}$.

Proof. The permutations $S_{1}$ of $\pi_{1}$ are the group $G$. By Theorem 5.3 the permutations of $\pi_{2}$ are $S_{2}=X^{-1} S_{1} Y$ for appropriate permutations $X$ and $Y$. Hence $S_{2}=X^{-1} G Y=X^{-1} G X \cdot X^{-1} Y$ is a left coset of $X^{-1} G X$, a transform of $G$. Also $S_{2}=X^{-1} Y \cdot Y^{-1} G Y$ is a right coset of $Y^{-1} G Y$.

Theorem 5.6(8). A permutation group $G$ on letters $c_{0}, \cdots, c_{n-1}$ such that

$(\alpha) G$ is doubly transitive,

$(\beta)$ only the identity fixes two letters,

$(\gamma)$ at most one element taking $c_{i}$ into $c_{j}$ displaces all letters, or

$\left(\gamma^{\prime}\right) n$ is finite

will be the permutations $S$ of a Euclidean plane $\pi^{*}$. The identity and the elements of $G$ which displace all letters form a simply transitive normal abelian subgroup $H$.

${ }^{(8)}$ When $n$ is finite, this theorem rests on a theorem of Frobenius, A. Speiser [1, p. 200], depending on the theory of group characters, or on the Sylow theorems as proved in R. D. Carmichael [1, p. 145]. Compare Lemma 8 with page 25 in G. Thomsen [1]. 
Proof. In a group, clearly conditions $(\alpha)$ and $(\beta)$ are equivalent to conditions (a) and (b) of Theorem 5.2. As is shown in appendix I, (a), (b) and ( $\left.\gamma^{\prime}\right)$ imply (c). When $n$ is infinite condition $(\gamma)$ is only half of condition (c). It is the "only one" part of the condition. We shall see in Lemma 5 below that the part "there exists one" of condition (c) is a consequence of $(\alpha)$ and $(\beta)$ for a group $G$. The second counter-example in appendix II shows that this is not a consequence of conditions (a) and (b). The author does not know whether $(\alpha)$ and $(\beta)$ for a group $G$ imply $(\gamma)$. A counter-example, if it exists, would have no particular geometric interpretation and would be of relatively little interest here. When Lemma 5 has been reached, we shall have shown that the permutations of $G$ are the permutations $S$ of a plane.

Lemma 1. There exists one and only one element of order two in $G$ which interchanges a pair of letters $(i, j)$.

Since $G$ is doubly transitive there exists an element $g=(\ldots, i, j, \ldots)$. If $h$ also interchanges $i$ and $j$ then $g h^{-1}$ fixes both $i$ and $j$, whence $g h^{-1}=1, g=h$. Hence $g$ is unique. Also $g^{2}$ fixes both $i$ and $j$ and hence $g^{2}=1$.

Lemma 2. The elements of order 2 in $G$ are a conjugate class.

An element of order 2 fixes at most one letter. If $n=2$ there is only one element of order 2 and the lemma holds. If $n \geqq 3$ then $g$ and $h$ of order 2 must both displace some letter $i ; g=(i j) \cdots, h=(i k) \cdots . G$ contains an element $x=\left(\begin{array}{l}i, j, \cdots \\ i, k, \cdots\end{array}\right)$ and $x^{-1} g x=(i k) \cdots=h$. If an element fixes a letter, then any conjugate will also fix some letter. Hence we may subdivide into two cases: Case I, The elements of order 2 displace all letters; Case II, Every element of order 2 fixes a letter.

Lemma 3. In Case II there is one and only one element of order 2 fixing a given letter.

In Case II, as before, $g=(i j) \cdots$ is transformed into $h=(i k) \cdots$ by $x=\left(\begin{array}{l}i, j, \cdots \\ i, k\end{array}, \ldots\right)$. But here if $g$ and $h$ both fix the letter $s$, then $x$ must also fix $s$. Hence $x \neq 1$ fixes both $i$ and $s$ which contradicts assumption $(\beta)$. If $g$ fixes a letter $s$ and $t$ is any other letter, an $x$ taking $s$ into $t$ will transform $g$ into an $h$ of order 2 which fixes $t$.

LEммa 4. The product of two different elements of order 2 is an element displacing all letters.

Suppose to the contrary that $g^{2}=1, h^{2}=1, g \neq h$, and $g h$ fixes the letter $i$. If either $g$ or $h$ fixes $i$ then the other must also, but this is impossible by Lemma 3. If both $g$ and $h$ displace $i$, then the letter $j$ into which $g$ takes $i$ must be taken into $i$ by $h$ and $g=(i j) \cdots, h=(j i)$, whence by Lemma $1 g=h$, contrary to assumption. Hence it is impossible that $g h$ could fix any letter. 
LEMMA 5. In $G$ there is at least one element, displacing all letters, which takes $a$ given $i$ into a given $j \neq i$.

In Case I there is an element of order 2, displacing all letters, which takes $i$ into $j$. In Case II there is an element $g$ of order 2 fixing $i$, and an element $h$ of order 2 taking $i$ into $j$. Here $g h$ takes $i$ into $j$ and by Lemma 4 displaces all letters.

As yet we have made no use of assumption $(\gamma)$. From now on it is necessary to use it. From Lemma 5 and $(\gamma)$ there is exactly one element in $G$, displacing all letters, taking $i$ into $j$. Now if $L$ is an element taking $k$ into $j$, $k \neq i$, then let $T$ be the element displacing all letters taking $i$ into $k$. Then take $M=T L$. Here $M$ takes $i$ into $j$ and $M L^{-1}=T$ displaces all letters. Since $T$ is unique, $M$ also is unique. This proves property (c). Hence the elements of $G$ form the permutations $S$ of a Euclidean plane $\pi^{*}$.

Lemma 6. In Case I every element displacing all letters is of order 2 and these together with the identity form a normal abelian subgroup.

Since there is an element of order 2, displacing all letters, taking $i$ into $j$, this must by $(\gamma)$ be the only element, displacing all letters, taking $i$ into $j$. But since $i$ and $j$ are arbitrary, every element displacing all letters must be of order 2. If $g^{2}=1, h^{2}=1$, then if $g=h, g h=1$, while if $g \neq h, g h$ displaces all letters and hence $(g h)^{2}=1$, whence $h g=g h$, and the elements of order 2 together with the identity form an abelian subgroup $H$. By Lemma $2, H$ is a normal subgroup. This completes the proof of the theorem for Case I.

LEMMA 7. In Case II an element $g$ displacing all letters may be written as the product $g=a b$ of two elements of order 2 where either $a$ or $b$ may be chosen arbitrarily.

Given $g$ displacing all letters and $a \neq 1, a^{2}=1$. Suppose that $a$ fixes the letter $i$ and that $g$ takes $i$ into $j$. Let $b=(i j) \cdots$. Then $a b$ takes $i$ into $j$ and by Lemma 4 displaces all letters, whence by $(\gamma), g=a b$. Similarly, given $g$ and $b$ fixing $k$, if $g$ takes $i$ in to $k$ and $a=(i k) \cdots$, then $g=a b$.

Lemмa 8. In Case II the product abc of three elements of order 2 is again an element of order 2, and $a b c=c b a$.

Here the lemma is trivial if $b=a$. If $b \neq a$, then $a b=g=d c$ where, by Lemma $7, d^{2}=1$. Hence $a b c=d c^{2}=d$. Since $d=d^{-1}, a b c=c^{-1} b^{-1} a^{-1}=c b a$.

Lemma 9. In Case II the elements displacing all letters, together with the identity, form a normal abelian simply transitive subgroup $H$.

By Lemmas 4 and 7 these are the elements of the form $a b$ where $a$ and $b$ are of order 2, and hence are a normal set. By Lemma 5 this set is transitive. Clearly the inverse of $a b$ is $b a$. To show that these form an abelian subgroup 
we must show $g_{1} g_{2}=1$ or $g_{3}$ and $g_{1} g_{2}=g_{2} g_{1}$. Here $g_{1} g_{2}=a_{1} b_{1} a_{2} b_{2}$ and by Lemma 7 $g_{1}=d a_{2}$, whence $g_{1} g_{2}=d b_{2}=1$ or $g_{3}$. By Lemma $8 g_{1} g_{2}=\left(a_{1} b_{1} a_{2}\right) b_{2}=\left(a_{2} b_{1} a_{1}\right) b_{2}$ $=a_{2}\left(b_{1} a_{1} b_{2}\right)=a_{2}\left(b_{2} a_{1} b_{1}\right)=g_{2} g_{1}$. This completes the proof of Theorem 5.6.

THEOREM 5.7. Under the assumptions of Theorem 5.6 in $\pi^{*}$ we have $x \cdot m \circ b=x m+b$ and addition and multiplication satisfy:

A1. $(a+b)+c=a+(b+c)$,

A2. $b+a=a+b$,

A3. $a+0=0+a=a$,

A4. $a+(-a)=(-a)+a=0$,

M1. $(a b) c=a(b c)$,

M3. $a 1=1 a=a$,

M4. $a a^{-1}=a^{-1} a=1$ if $a \neq 0$,

M5. $a 0=0 a=0$,

D1. $(a+b) m=a m+b m$,

E3. $x r=x s+t$ has a unique solution $x$ if $r \neq s$.

Proof. Following Theorem 5.4 the identity is $I=\left(\begin{array}{c}0,1, \ldots \\ 0,1, \ldots\end{array}\right)$. If $A_{a}=\left(\begin{array}{l}0, \cdots, u, \cdots \\ a, \cdots, 0, \cdots\end{array}\right)$ is the permutation taking 0 into $a$ and displacing all letters then $v=u \cdot 1 \circ a=u+a$. If $M_{m}=\left(\begin{array}{l}0,1, \cdots, w, \cdots \\ 0, m, \cdots, 2, \cdots\end{array}\right)$ then $z=w \cdot m \circ 0=w m$. An arbitrary permutation may be written in the form $L=\left(\begin{array}{l}0,1, \cdots \\ b,\end{array}, \cdots\right)$ $=\left(\begin{array}{c}0,1, \cdots \\ 0, m, \cdots\end{array}\right)\left(\begin{array}{c}0, \cdots, m, \cdots \\ 0, \cdots, c, \cdots\end{array}\right)=M_{m} \cdot A_{b}$ whence $x \cdot m \circ b=x m+b$.

From Theorem 5.6 the $A_{a}$ 's and $I$ form an abelian subgroup $H$ whence $\mathrm{A} 1, \mathrm{~A} 2, \mathrm{~A} 3$, and A4 hold. The $M_{m}$ 's and $I$ form the subgroup of $G$ fixing the letter $O$, whence multiplication forms a group and M1, M3, M4 hold. To show that the one-sided distributive law D1 holds we make use of the fact that $H$ is a normal subgroup in $G$. Let $A_{a}=\left(\begin{array}{c}0, \cdots \\ a, \cdots\end{array}\right), A_{b}=\left(\begin{array}{l}0, \cdots, \\ b, \cdots, a+b, \cdots\end{array}\right)$, $M_{m}=\left(\begin{array}{c}0,1, a, b, \ldots \\ 0, m, a m, b m, \cdots\end{array}\right)$. Here $M_{m}^{-1} A_{a} M_{m}=\left(\begin{array}{c}0, \cdots \\ a m, \cdots\end{array}\right)=A_{a m}$ since all letters are displaced. Also $M_{m}^{-1} A_{b} M_{m}=A_{b m}$. Hence $A_{(a+b) m}=M_{m}^{-1} A_{a+b} M_{m}$ $=M_{m}^{-1} A_{a} A_{b} M_{m}=M_{m}^{-1} A_{a} M_{m} \cdot M_{m}^{-1} A_{b} M_{m}=A_{a m} A_{b m}=A_{a m+b m}$, whence $(a+b) m$ $=a m+b m$. The other distributive law $a(r+s)=a r+a s$ will not in general be true. The law E3 is an immediate consequence of property (5) of Theorem 5.4 and the fact that $x \cdot m \circ b=x m+b$ in this theorem.

We may modify the notation for points and lines in $\pi^{*}$ and adjcir $L_{\infty}$ and the points on it to obtain a pseudo-homogeneous representation for the projective plane $\pi$. Let $P^{*}=(x, y)$ in $\pi^{*}$ be written $P=(x,-y, 1)$ in $\pi$. If the line $y=x m+b$ in $\pi^{*}$ cuts $L_{\infty}$ in $\pi$ in the point $Q$, write $Q=(1,-m, 0)$ in $\pi$. Finally write $A=(0,1,0)$. Then the lines $y=x m+b$ in $\pi^{*}$ become $y+x m+z b$ $=0$ containing points $(x, y, z)$ in $\pi$. Also write $x=c$ in $\pi^{*}$ as $x+z(-c)=0$ in $\pi$, and finally $L_{\infty}$ is $z=0$. The associative law of multiplication and D1 permit us to take any equation $y a+x b+z c=0$ as the equation of a line, but without the distributive law D2: $a(b+c)=a b+a c$ we cannot write the points in homogeneous form. The associative law of multiplication, M1, is not necessary in order that a ring satisfying the remaining axioms of Theorem 5.7 define a 
plane, and such rings and planes will be investigated below. It is to include such rings and planes in the above transition from an affine to a projective plane that the apparently unnecessary step of changing the sign of the $y$-coordinate is made, since from the remaining axioms it does not follow that $-x m=x(-m)$ although it is true that $-x m=(-x) m$.

Veblen and Wedderburn $\left({ }^{9}\right)$ have constructed certain number systems suitable for the coordinatization of planes very closely related to the rings characterized in Theorem 5.7. A more thorough investigation of these number systems and the planes which they define is given here. In $\$ 6$ it will be shown that these systems correspond to a particular configuration theorem and further properties of these planes will be considered.

THEOREM 5.8. Given a ring $R$ in which addition and multiplication satisfy:

A1. $(a+b)+c=a+(b+c)$,

A2. $b+a=a+b$,

A3. $a+0=0+a=a$,

A4. $a+(-a)=(-a)+a=0$,

M5. $0 a=a 0=0$,

D1. $(a+b) c=a c+b c$,

E1. $x a=b, a \neq 0$, has $a$ unique solution $x$,

E2. $a z=b, a \neq 0$, has a unique solution $z$,

E3. $x r=x s+t, r \neq s$, has a unique solution $x$.

Then if $\phi \neq 0$ and $\psi \neq 0$ are fixed but arbitrary in $R$ we may define points $(x, y, z)$ of forms $(x, y, \phi),(\phi, y, 0),(0, \phi, 0)$ where $x$ and $y$ take all values in $R$. Lines are defined as those sets of points satisfying one of the equations $y \psi+x b+z c$ $=0, x \psi+z c=0, z \psi=0$, where $b$ and $c$ take all values in $R$. Points and lines so defined form a projective plane $\left({ }^{10}\right) \pi$.

The plane $\pi$ is independent of the particular choice of $\phi$ and $\psi$. We may define a new multiplication o by the rule

$$
u=v \circ s \text { if and only if } s \psi=\phi \lambda, \quad u \psi=v \lambda .
$$

The operations,+ , define a ring $R_{1}$ satisfying all the properties which $R$ does and determining the same plane. $R_{1}$ has the additional property that $\phi$ is a unit:

$$
M 3, \phi \circ a=a \circ \phi=a \text {. }
$$

Proof. We must first show that there is one and only one line through two distinct points $P$ and $Q$. We must treat cases separately according to the

(9) O. Veblen and J. H. M. Wedderburn [1].

${ }^{\left({ }^{10}\right)}$ The systems used by O. Veblen and J. H. M. Wedderburn are slightly different. Here the right distributive law is used rather than the left, and the roles of the $x$ and the $y$ are reversed. To go from their planes to these given here we consider the rings anti-isomorphic to theirs, take the dual plane, and then interchange the $x$ and $y$. They do not state the analogue of the necessary condition E3, nor do they observe that the plane is independent of the choice of $\phi$ and $\psi$. 
different forms for $P$ and $Q$. Every case goes through almost trivially except for $P=\left(x_{1}, y_{1}, \phi\right), Q=\left(x_{2}, y_{2}, \phi\right)$, and here with $x_{1} \neq x_{2}, y_{1} \neq y_{2}$. Neither $P$ nor $Q$ lies on $z \psi=0$ and we cannot have $x_{1} \psi+z c=0$ and $x_{2} \psi+z c=0$ with $x_{1} \neq x_{2}$. Hence the line $P Q$, if there is one, is of the form $y \psi+x b+z c=0$. Now observe from D1, putting $b=-a$, that $0=0 m=a m+(-a) m$ whence $-a m=(-a) m$. Hence if $y_{1} \psi+x_{1} b+\phi c=0$ and $y_{2} \psi+x_{2} b+\phi c=0$, by subtraction and D1 $\left(y_{1}-y_{2}\right) \psi+\left(x_{1}-x_{2}\right) b=0$ whence $\left(x_{1}-x_{2}\right) b=\left(-y_{1}+y_{2}\right) \psi$. From E1 this determines a unique $b$. Then from $\mathrm{E} 2, \phi c=-y_{1} \psi-x_{1} b$ determines $c$ uniquely. From this determination of $b$ and $c$ we find a unique line containing $P$ and $Q$. To show that two different lines intersect in one and only one point we must again treat several cases according to the form of the lines. These all go through quite easily except when they are of the forms $y \psi+x b_{1}+z c_{1}=0$, $y \psi+x b_{2}+z c_{2}=0$, and here only when $b_{1} \neq b_{2}$. Since $\psi \neq 0$ it cannot be a point $(0, \phi, 0)$ or $(\phi, y, 0)$ with $b_{1} \neq b_{2}$. Hence it must be a point $(x, y, \phi)$. Substituting $z=\phi$ we have $x b_{1}=x b_{2}+\phi c_{2}-\phi c_{1}$ and by E3, since $b_{1} \neq b_{2}$, there is a unique solution $x$. Then $y \psi=-x b_{1}-z c_{1}$ by E1 determines $y$ uniquely and we find a unique point $(x, y, \phi)$ on both lines. Hence these points and lines form a projective plane $\pi$.

To show that $\pi$ is independent of the particular choice of $\phi$ and $\psi$, we show first that for $\phi$ fixed it is independent of $\psi$, and then that for $\psi$ fixed it is independent of $\phi$.

Let $\pi_{1}$ be the plane determined by $\phi$ and $\psi_{1}$, and $\pi_{2}$ be the plane determined by $\phi$ and $\psi_{2}$. The relation $u \psi_{1}=u^{\prime} \psi_{2}$ determines by E1 a 1-1 mapping $u \rightleftarrows u^{\prime}$ of $R$ into itself leaving 0 fixed. Also let $\phi c=-a \psi_{1}, \phi d=-a \psi_{2}$ determine the mapping $c \rightleftarrows d$. The correspondences

$$
\begin{aligned}
(x, y, \phi) & \rightleftarrows\left(x, y^{\prime}, \phi\right), \\
(\phi, y, 0) & \rightleftarrows\left(\phi, y^{\prime}, 0\right), \\
(0, \phi, 0) & \rightleftarrows(0, \phi, 0), \\
y \psi_{1}+x b+z c=0 & \rightleftarrows y \psi_{2}+x b+z c=0, \\
x \psi_{1}+z c=0 & \rightleftarrows x \psi_{2}+z d=0, \\
z \psi_{1}=0 & \rightleftarrows z \psi_{2}=0,
\end{aligned}
$$

set up an isomorphism between $\pi_{1}$ and $\pi_{2}$. Hence $\pi$ is independent of the choice of $\psi$.

Now let $\pi_{1}$ be the plane determined by $\phi_{1}$ and $\psi$ and $\pi_{2}$ be determined by $\phi_{2}$ and $\psi$. By E2, $\phi_{1} u=\phi_{2} u^{\prime}$ determines a 1-1 mapping of $R$ onto itself leaving 0 fixed. Also let $y \rightleftarrows \tilde{y}$ be determined by $y \psi=-\phi_{1} b,-\phi_{2} b=\tilde{y} \psi$. Then the correspondences

$$
\begin{aligned}
&\left(x, y, \phi_{1}\right) \rightleftarrows\left(x, y, \phi_{2}\right), \\
&\left(\phi_{1}, y, 0\right) \rightleftarrows\left(\phi_{2}, \tilde{y}, 0\right), \\
&\left(0, \phi_{1}, 0\right) \rightleftarrows\left(0, \phi_{2}, 0\right),
\end{aligned}
$$




$$
\begin{aligned}
y \psi+x b+z c & =0 \rightleftarrows y \psi+x b+z c^{\prime}=0, \\
x \psi+z c & =0 \rightleftarrows x \psi+z c^{\prime}=0, \\
z \psi & =0 \rightleftarrows z \psi=0,
\end{aligned}
$$

are an isomorphism between $\pi_{1}$ and $\pi_{2}$.

Now let us form a new ring $R_{1}$ whose elements are the same as those of $R$. In $R_{1}$ addition is the same as in $R$, but multiplication is a new operation (0) as defined by (5.5), using arbitrary but fixed values for $\phi$ and $\psi$. In $R_{1}$ A1, 2, 3, 4 are clearly satisfied since the operation of addition is the same as in $R$. M5 for $R_{1}$ comes immediately from (5.5). Let $a \circ m=w, b \circ m=z$, whence, by (5.5), $m \psi=\phi c, a \psi=w c, b \psi=z c$. Adding the last two of these and using D1 for $R$ we have $(a+b) \psi=(w+z) c$, whence, by (5.5), $(a+b) \circ m=w+z=a \circ m$ $+b \circ m$, proving D1 for $R_{1}$. Now $x \circ a=b$ with $a \neq 0$ is equivalent to $a \psi=\phi c$ and $b \psi=x c$, where the first determines $c \neq 0$ uniquely and the second determines $x$ uniquely, proving E1 for $R_{1}$. Similarly E2 for $R_{1}$ follows immediately. If $x \circ r=x \circ s+t, r \neq s$, we have with $x \circ r=z, x \circ s=w$,

$$
\begin{aligned}
z=w+t, & r \psi & =\phi m, \quad s \psi=\phi n, \\
z \psi=x m, \quad w \psi=x n, & z \psi & =w \psi+t \psi, \quad x m=x n+t \psi .
\end{aligned}
$$

Since $r \neq s, m$ is different from $n$, and so by E3 for $R$ the last equation determines $x$ uniquely. This proves property E3 for $R_{1}$. This shows that $R_{1}$ satisfies all the properties which $R$ does. Also in $R_{1}$ if $a \circ \phi=b$, then $\phi \psi=\phi m$, $b \psi=a m$, whence $m=\psi$ and $b=a$. If $\phi \circ a=b$ then $a \psi=\phi m, b \psi=\phi m$, whence $a \psi=b \psi$ and $a=b$. Hence in $R_{1}$ the rule M3, $\phi \circ a=a \circ \phi=a$, is satisfied.

It remains to show that $R_{1}$ defines the same plane $\pi$ which $R$ defines. For this purpose we shall take $\phi$ and $\psi$ from $R$ to determine the plane and $\phi$ and $\phi$ from $R_{1}$. Remembering that in $R_{1}$ we may write $\phi$ as 1 , we see that the isomorphism is

$$
\begin{aligned}
R & \rightleftarrows R_{1}, \\
(x, y, \phi) & \rightleftarrows(x, y, 1), \\
(\phi, y, 0) & \rightleftarrows(1, y, 0), \\
(0, \phi, 0) & \rightleftarrows(0,1,0), \\
y \psi+x b+z c=0 & \rightleftarrows y+x \circ b^{\prime}+z \circ c^{\prime}=0, \\
x \psi+z c=0 & \rightleftarrows x+z \circ c^{\prime}=0, \\
z \psi=0 & \rightleftarrows z=0,
\end{aligned}
$$

where $c^{\prime} \psi=\phi c, b^{\prime} \psi=\phi b$. Note that the isomorphism does not alter the coordinates of points. If we use the correspondence $s \rightleftarrows s^{\prime}$, where $s^{\prime} \psi=\phi s$, then from (5.5) we have immediately $\left(v \circ s^{\prime}\right) \psi=v s$. Multiplying the line equations for $R_{1}$ on the right by $\psi$ and using this rule, we see immediately that they are equivalent to the line equations from $R$ just as the correspondence is given in (5.10). 
This process is of course reversible in going from line equations from $R$ to those from $R_{1}$. This establishes the isomorphism, and completes the proof.

THEOREM 5.9. If a plane $\pi$ is determined by a ring $R$ satisfying axioms $\mathrm{A} 1,2,3,4, \mathrm{M} 3,5, \mathrm{D} 1$, and $\mathrm{E} 1,2,3$ as in Theorem 5.8 then $R$ is the ternary ring determined by $\pi$ as in Theorem 5.4 , where $x \cdot m \circ b=x m+b$, taking $A, B,(0,0,1)$ and $(0,-1,1)$ as the fundamental quadrilateral. The correspondence between the two representations is given by

$$
\begin{aligned}
A & \rightleftarrows A, \\
B & \rightleftarrows B, \\
(0,0) & \rightleftarrows(0,0,1), \\
(1,1) & \rightleftarrows(1,-1,1), \\
x=c & \rightleftarrows x+(-c)=0, \\
y=x m+b & \rightleftarrows y+x m+b=0 .
\end{aligned}
$$

The mapping $(x, y) \rightarrow(x+s, y+t)$ for arbitrary $s, t \subset R$ is a collineation of $\pi$ leaving the points of $L_{\infty}$ fixed. If a plane $\pi_{1}$ is determined by such a ring $R_{1}$ and $\pi_{2}$ is any plane isomorphic to $\pi_{1}$ with $A_{1} \rightleftarrows A_{2}, B_{1} \rightleftarrows B_{2}$ then in the ring $R_{2}$ of $\pi_{2}, x \cdot m \circ b=x \circ m+b$ where the addition ( + ) and multiplication (o) satisfy' axioms $\mathrm{A} 1,2,3,4, \mathrm{M} 3,5, \mathrm{D} 1$, and $\mathrm{E} 1,2,3$. Moreover $R_{2}$ may be considered as containing the same elements as $R_{1}$ where addition is the same and multiplication (o) may be determined from that of $R_{1}$ by the rule

$$
w=x \circ d \text { if and only if } d s=a t, w s=x t,
$$

for appropriate fixed $a \neq 0, s \neq 0$, and all $x$ and $d$.

Proof. Given a ring $R$ with unit determining a plane as in Theorem 5.8. The correspondences (5.11) determine points $(x, y)$ and lines $x=c, y=x m+b$ satisfying the postulates for a Euclidean plane isomorphic to the finite part of the projective plane given by the pseudo-homogeneous coordinatization from $R$. Let us now introduce coordinates in this plane according to the rules of Theorem 5.4 with $A, B,(0,0)$ and $(1,1)$ as the fundamental quadrilateral. A line $y=x m$ goes through $(0,0)$ and $(1, m)$ and hence is the line $y=x \cdot m \circ 0$. The line $y=x m+b$ with $b \neq 0$ is parallel to $y=x m$ and goes through $(0, b)$ and hence is $y=x \cdot m \circ b$. Here lines $x=c$ are clearly the same in both representations. Hence $x \cdot m \circ b=x m+b$ and $R$ is the ring determined by $\pi$ as in Theorem 5.4.

The mapping $(x, y) \rightarrow(x+s, y+t)$ takes the points of the line $x=c$ into those of $x=c+s$ and the points of $y=x m+b$ in to those of $y=x m+(s m+b-t)$ whence it is a collineation leaving the slopes of lines unchanged and hence leaving the points of $L_{\infty}$ fixed. As $s$ and $t$ are arbitrary these collineations are simply transitive on the finite points of $\pi$.

Given a plane $\pi_{1}$ determined by a ring $R_{1}$, let $\pi_{2}$ be a plane isomorphic 
to $\pi_{1}$ with $A_{1} \rightleftarrows A_{2}, B_{1} \rightleftarrows B_{2}$. Since there are collineations of $\pi_{1}$ transitive on its finite points and leaving $A$ and $B$ fixed there will be such an isomorphism which also maps $(0,0)$ of $\pi_{1}$ onto $(0,0)$ of $\pi_{2}$. Hence for an appropriate $s$, $y=x s$ of $\pi_{1}$ is mapped onto $y=x$ of $\pi_{2}$, and we have $(x, x s) \rightarrow(u, u)$. Now replace the symbols used in $\pi_{2}$ so that this mapping becomes $(x, x s) \rightarrow(x, x)$. Hence in general, mapping $\pi_{1}$ onto $\pi_{2}$,

$$
(x, y s) \rightarrow(x, y) \text {. }
$$

Now consider the natural ring $R_{\mathbf{2}}$ of $\pi_{2}$ (the ternary ring of Theorem 5.4) determined by the fundamental quadrilateral $A, B, O=(0,0)$ and $I=(a, a)$. Note that the symbols used are taken from $\pi_{1}$ and that the unit 1 of $\pi_{1}$ need not be the unit $a$ of $\pi_{2}$, although their respective origins coincide. A line $y=x \cdot a \circ c$ is the image of a line $y=x s+t$ where $(0, t) \rightarrow(0, c)$ whence $t=c s$ and a point $(x, y)$ on it is the image of $(x, y s)$. Hence $y=x+c$ in $R_{2}$ if and only if $y s=x s+t=x s+c s=(x+c) s$ in $R_{1}$, whence $y=x+c$ in $R_{1}$ and so addition in $R_{2}$ is the same operation as addition in $R_{1}$. In $R_{2}, y=x \cdot d \circ 0$ goes through $(0,0)$ and $(a, d)$ and so is the image of the $R_{1}$ line through $(0,0)$ and $(a, d s)$, which is $y=x t$ where $d s=a t$ determines $t$. Hence if $w=x \circ d$ in $R_{2}$ then $(x, w)$ in $\pi_{2}$ is the image of $(x, w s)$ in $\pi_{1}$ where $w s=x t$. Hence $d s=a t$, ws $=x t$ in $R_{1}$ define $w=x \circ d$ in $R_{2}$, and this is the rule (5.12). In $\pi_{2}$ the line $y=x \cdot m \circ b$ goes through $(0, b)$ and is parallel to $y=x \circ m$ which goes through $(0,0)$ and $(a, m)$. These are the images of $(0,0)$ and $(a, m s)$ in $\pi_{1}$. Hence it is the image of $y=x t$ where $a t=m s$. Also $(0, b s) \rightarrow(0, b)$ and so $y=x t+b s \rightarrow y=x \cdot m \circ b$. Hence by (5.12) $y=x t+b s \rightarrow y=x \circ m+b$, and so in $R_{2}, x \cdot m \circ b=x \circ m+b$. We may now verify, as in Theorem 5.7, that $R_{2}$ has properties $\mathrm{A} 1,2,3,4$, M3, 5, E1, 2, 3 where $a$ is the unit of $R_{2}$.

TheOREM 5.10( $\left.{ }^{11}\right)$. Rings $R_{1}$ and $R_{2}$ with properties $\mathrm{A}, \mathrm{M}, \mathrm{D}, \mathrm{E}$ of Theorem 5.9 determine planes $\pi_{1}$ and $\pi_{2}$ isomorphic with $A_{1} \rightleftarrows A_{2}, B_{1} \rightleftarrows B_{2}$ if and only if each can be obtained from the other by rule (5.12). If in $R_{1}$ multiplication is

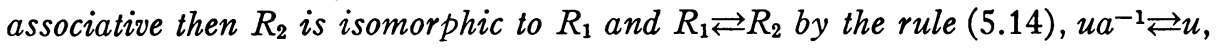
$u a^{-1}+v a^{-1} \rightleftarrows u+v,\left(u a^{-1}\right)\left(v a^{-1}\right) \rightleftarrows u \circ v$.

Proof. From Theorem 5.9, $R_{1}$ and $R_{2}$ are each determined by their planes $\pi_{1}$ and $\pi_{2}$ and the choice of the fundamental quadrilateral $A, B, O, I$. If $A_{1} \rightleftarrows A_{2}$, $B_{1} \rightleftarrows B_{2}, R_{2}$ may be obtained according to Theorem 5.9 from $R_{1}$ with the same addition and with multiplication given by the rule (5.12). Similarly $R_{1}$ may be obtained from $R_{2}$. If in $R_{1}$ multiplication is associative, from $d s=a t$, ws $=x t$ we have $t=a^{-1} d s$, ws $=x a^{-1} d s, w=x a^{-1} d$ whence $x \circ d=x a^{-1} d$. Using this we easily verify the correspondences (5.14). Note that if multiplication is associative, then the substitutions $x \rightarrow x m+b, m \neq 0$, form a doubly transitive group as in Theorem 5.6.

(11) If $R_{1}$ does not have an associative multiplication $R_{2}$ need not be algebraically isomorphic to $R_{1}$. See appendix II for examples. 
We shall summarize the results of this section briefly. For every choice of a pair of points $A, B$ in a plane $\pi$ a set $S$ of doubly transitive permutations is determined (Theorem 5.2) and if $L, M$ are two of these, $M L^{-1}$ is associated with a projection in $\pi$. Conversely a doubly transitive set $S$ of permutations satisfying the necessary conditions determines a plane $\pi$ (Theorem 5.2). Every choice of a quadrilateral $A, B, O, I$ in $\pi$ determines a ternary ring $R$ (Theorem 5.4) with a zero and a unit. If $\pi$ is an analytic plane over a skewfield $F$ then $R=F$ with $x \cdot m \circ b=x m+b$ for every choice of $A, B, O, I$ (equations 5.2, Theorem 5.9). A plane represented by a Veblen-Wedderburn number system is independent of the choice of $\phi$ and $\psi$, and may also be represented by a Veblen-Wedderburn number system with a unit (Theorem 5.8), and this system is one of the rings $R$ determined by $\pi$ as in Theorem 5.4 (Theorem 5.9). Multiplication in this system is associative if and only if the $A-B$ permutations $S$ form a group (Theorems $5.6,5.7,5.10$ ) or a coset of a group (Theorem 5.5).

6. Configuration theorems. A configuration theorem is a statement of the following sort: Given points $P_{1}, \cdots, P_{m}$ and lines $L_{1}, \cdots, L_{n}$ and the information that (1) at least certain points $(P)$ are distinct; $(2)$ at least certain lines $(L)$ are distinct; (3) $P_{i} \subset L_{j}$ for certain points and lines; and (4) $P_{r} \subset L_{s}$ for certain points and lines, then we may conclude that there are further incidences $P_{u} \subset L_{v}$.

Axiom A3 of Veblen and Young is of this type as are also the Theorems of Desargues and Pappus. It is natural to enquire what theorems of this sort are possible and what relationships exist between such theorems. Outstanding in this connection is Hessenberg's proof that the Theorem of Desargues is a consequence of the Theorem of Pappus $\left({ }^{12}\right)$. Moufang $\left({ }^{13}\right)$ has investigated a number of theorems which are consequences of the Desargues Theorem and in particular has shown that the Theorem of the complete quadrilateral is definitely weaker than the Theorem of Desargues. For the most part Moufang assumes not only Hilbert's axioms of connection but also the axioms of order. Most of her results are, however, true without the hypothesis of order.

In a configuration theorem, we are given a configuration $C$ and the conclusion leads to a configuration $C^{*}$ containing the points and lines of $C$ and further incidences. In the statement of a configuration theorem above, $C^{*}$ was assumed to have exactly the same points and lines as $C$. It is an unimportant generalization to permit $C^{*}$ to contain points and lines constructed from $C$. If the given configuration is not open then Moufang calls the theorem conditional. If $C$ is open and $C^{*}$ is obtained by adding a single incidence to $C$,

(12) G. Hessenberg [1]

(13) R. Moufang [1-7]. She has shown in [6] that the validity of the theorem of the complete quadrilateral is equivalent to the possibility of introducing coordinates from an alternative field, and that there exist alternative fields (that is, the Cayley algebra) which are not skewfields. Alternative fields are covered in M. Zorn [1, 2] and in R. Moufang [7]. 
we shall call the theorem simple. Most theorems may be treated as a combination of several simple theorems. $C$ is free equivalent by Theorem 4.9 to some $r$-point (4.3) and if $C^{*}$ is also open it is free equivalent to some $(r-1)$ point, since in Theorem 4.11 $P$ and $L$ are unchanged and $I$ has been increased by 1 . This means that any finite configuration in the plane can be generated by an $(r-1)$-point and in a restricted way. All such theorems which the author has investigated are demonstrably impossible. We shall restrict our attention to theorems where $C$ is open and $C^{*}$ is confined. For if $C^{*}$ is not open then it contains a confined configuration which may be obtained by free contraction from $C^{*}$ and this may be considered the consequence of the appropriate incidences in part of $C$.

As a further refinement in the classification of configuration theorems we distinguish between theorems in which the points and lines of $C$ are arbitrary, apart from the mutual relationships required of them, and theorems in which certain points and lines of $C$ are fixed. The first type we shall call a universal theorem, the second a fixed point theorem. Hilbert's formulation of the Desargues Theorem is a fixed point theorem in that he requires the axis of perspectivity always to be the line at infinity. Theorems on webs $\left({ }^{14}\right)$ are of course fixed point theorems.

The Theorem of Pappus in a plane $\pi$ is equivalent to the possibility of introducing coordinates from a commutative field $F$. What further configuration theorems can there be in such a plane $\pi$ ? Given $C$ we may take four of its generating points as a fundamental quadrilateral and introduce $F$ as the natural ring of $\pi$ determined by this quadrilateral. The other points and lines of $C$ may be represented by values from $F$, including parameters representing the arbitrariness of the choice of the generators of $C$. The conditions (1), (2), (4) will correspond to a relation $A(u, \cdots, w) \neq 0$ where $A$ is an appropriate rational function of the parameters $u, \cdots, w$. After we express the coordinates of the points and the equations of the lines in terms of the parameters the conclusion $P \subset L$ will determine a relation $B(u, \cdots, w)=0$. Hence the theorem may be stated thus: $B=0$ whenever $A \neq 0$, whence $A B=0$ for all values for which $A B$ is defined. If $A B=M / N$ where $M$ and $N$ are polynomials, then $M N=0$ for all values of the parameters. Here $M N$ is a polynomial, simplified only according to the general rules of fields and not according to special properties of $F$, such as finite characteristic or particular relations between fixed elements of $F$. If $M N$ is formally zero, then the theorem is a consequence of the field properties of $F$ and hence of the Theorem of Pappus. If $M N$ is not formally zero and involves no parameters, then the theorem either implies that $F$ has finite characteristic or expresses a relation on fixed elements of $F$.

(14) W. Blaschke and G. Bol [1] is an excellent work on the subject of webs and includes an extensive bibliography. A long series of papers on Topologische Fragen der Differentialgeometrie, beginning in 1927, mostly appearing in the Abh. Math. Sem. Hamburgischen Univ., are largely concerned with web configurations. 
In the latter case the entire configuration is fixed. If $M N$ contains parameters $x_{1}, \cdots, x_{r}$ then $M N=a_{0}\left(x_{1}, \cdots, x_{r-1}\right) x_{r}^{t}+\cdots+a_{t}\left(x_{1}, \cdots, x_{r-1}\right)$. In this case $F$ must be a finite field. For either $a_{0}\left(x_{1}, \cdots, x_{r-1}\right)=0$ for all $x_{1}, \cdots, x_{r-1}$ of $F$ or there is a polynomial $a_{0} x_{r}^{t}+\cdots+a_{t}$, which is not identically zero, which vanishes for all $x_{r} \subset F$. In either event we are led eventually to a nonzero polynomial in one argument $x$ which vanishes for all choices of $x$ from $F$. But such a polynomial has only a finite number of roots and hence $F$ must be a finite field. In the finite field with $n=p^{8}$ elements every element satisfies $x^{n}-x=0$. Our conclusion will be stated as a theorem:

THEOREM 6.1. If the Theorem of Pappus is valid in a plane $\pi$, then any configuration theorem which does not consist entirely of fixed points is either (1) a consequence of the Theorem of Pappus, (2) equivalent to characteristic $p$ for $F$, or (3) equivalent to finileness for $F$ and hence for $\pi$.

In a plane $\pi$ if the Desargues Theorem is valid but not the Pappus Theorem, then corresponding to any configuration theorem in $\pi$ not a consequence of the Desargues Theorem, there is an identity in the coordinatizing skew-field $K$. A universal theorem in $\pi$ will correspond to an identity expressible entirely in terms of the unit 1 and parameters. Here there is no canonical form for rational functions of more than one indeterminate and little is known about the possibility of universal identities $\left({ }^{15}\right)$. If there is a one-parameter universal identity in $K$, it can, as above, be shown to be equivalent to a polynomial identity with coefficients from the characteristic subfield. Hence, as $0,1, \ldots$ must be roots, the characteristic must be a finite $p$. If this identity is $F(x)=0$ where $F(x)$ is a polynomial which does not vanish identically, then we may suppose without loss of generality that $F(x)$ has no multiple factors. In this case if $n$ is the least common multiple of the degrees of the divisors of $F(x)$ then, from well known theorems on polynomial decomposition modulo $p, F(x)$ is a divisor of $x^{N}-x$, where $N=p^{n}$. Hence $x^{N}=x$ for every $x$ in $K$, and $x^{N-1}=1$ for every $x \neq 0$ in $K$. For $x \neq 0, x^{-1}=x^{N-2}$ and so the subfield generated by elements $x, y, \cdots, z$ is a polynomial ring in these elements. Consider the subfield $Q(x, y)$. Every element in $Q$ is a linear combination with coefficients $0,1, \cdots, p-1$ of elements from the group $G(x, y)$ generated by $x$ and $y$. Every element in $G$ has order dividing $N-1$. If Burnside's conjecture is correct $G$ must be finite $\left({ }^{16}\right)$. If this conjecture is true, then $Q$ must be finite, and by the Wedderburn Theorem $Q$ is a Galois field and $y x=x y$. Hence $K$ is commutative, whence $K$ itself is a finite field containing at most

(15) In D. E. Littlewood [1] and A. R. Richardson [1] polynomial relations with coefficients from $K$ are considered. W. Wagner [1] considers universal polynomial relations and shows that none exist in ordered planes, but that there always exist universal identities in division algebras, since the finite basis permits a matric representation and matric identities exist. B. H. Neumann [1] and others have treated the analogous problem for groups.

${ }^{(16)}$ This conjecture is discussed in B. H. Neumann [1]. Analogous ring and field problems are discussed in A. Kurosch [1]. 
$p^{n}$ elements. But even if this conjecture on groups is false it may very well be true that $x^{N}=x, N=p^{n}$ for all elements of a skew-field $K$, implies that $K$ is finite (and hence is a Galois field with at most $p^{n}$ elements). For $n=1$, $F(x)=x(x-1) \cdots(x-p+1)$ and $K$ is the characteristic field $K(p)$. For $n=2$ every element of $K$ not in $K(p)$ satisfies an equation of second degree over $K(p)$. Hence $x^{2}=a x+b, y^{2}=c y+d$, and $x^{2}$ and $y^{2}$ are expressible as functions of first degree. Also $(x+y)^{2}=g(x+y)+h$ and $x^{2}+x y+y x+y^{2}=1$ st degree, whence $y x=-x y+1$ st degree. Here every third degree expression in $x$ and $y$ is also expressible as one of at most second degree. For example $x y x=x(-x y+1$ st degree $)=-x^{2} y+2$ nd degree $=(1$ st degree $) y+2$ nd degree $=2$ nd degree. Hence every expression of third degree or higher can be reduced to second degree, and $1, x, y, x y$ are a basis of $Q(x, y)$ whence $Q$ is finite and $K$ is finite and contains at most $p^{2}$ elements.

For $n=3$ let us suppose first that $p \neq 2$. We have $x^{3}, y^{3},(x+y)^{3},(x-y)^{3}$ all of second degree. Combining these we get

$$
\begin{aligned}
x^{2} y+x y x+y x^{2}+x y^{2}+y x y+y^{2} x & =2 \text { nd degree, } \\
-x^{2} y-x y x-y x^{2}+x y^{2}+y x y+y^{2} x & =2 \text { 2nd degree, }
\end{aligned}
$$

whence since $p \neq 2$

$$
\begin{aligned}
& x^{2} y+x y x+y x^{2}=2 \text { nd degree, } \\
& x y^{2}+y x y+y^{2} x=2 \text { nd degree. }
\end{aligned}
$$

Using these we need retain for a basis of $Q$ only $x^{2} y, x y x, x y^{2}, y x y$ of third degree, and $x^{2} y x, x^{2} y^{2}, x y x y, y x y x$ of fourth degree. Using $(x y)^{3},(y x)^{3},(x y+x)^{3}$, $(y x+y)^{3}$ as of fourth degree, we find that $x y x y x$ and $y x y x y$ are of fourth degree. Hence of fifth degree terms we need retain only $x^{2} y x y$ and $x y x y^{2}$, and of sixth only $x^{2} y x y^{2}$, and no terms at all of seventh or higher degree. Hence $Q$ is finite and $K$ is finite with at most $p^{3}$ elements. A similar but slightly longer argument holds for $p^{3}=2^{3}=8$. On the basis of these computations and other considerations the author makes the conjecture that in a Desarguesian plane there can be a universal five point theorem which is not a consequence of the Desargues theorem only if the plane is finite.

A universal theorem on six or more points corresponds to a universal identity with two or more parameters. As noted above no such theorems exist in an ordered plane, but theorems certainly exist in planes coordinatized by division algebras (skew-fields with a finite basis over their center). In any quaternion algebra $\left({ }^{17}\right)$ if $x, y, z$ are three arbitrary elements, then $z(x y-y x)^{2}$ $=(x y-y x)^{2} z$, or in words, the square of any commutator is in the center. This identity corresponds to a seven point universal theorem in Desarguesian planes. To find the fullest algebraic consequences of this geometric theorem, we prove the following theorem.

${ }^{(17)}$ For the general quaternion algebra, see A. A. Albert [1, p. 145]. 
Theorem 6.2. Given a skew-field $K$ whose center is $F$. If $z(x y-y x)^{2}$ $=(x y-y x)^{2} z$ for three arbitrary elements of $K$, then either $K=F$ or $K$ is a quaternion algebra over $F$.

Proof. Here the square of every commutator $x y-y x$ is in $F$.

Lemma 1. An arbitrary element $x$ of $K$ not in $F$ satisfies an equation $x^{2}=r x+s$ with $r, s \subset F$.

The proof is relatively simple if the characteristic of $K$ is not 2 . Here let $y$ be an element which does not permute with $x$ and write $u=y x-x y$, $u^{2}=a \subset F, a \neq 0, v=y x^{2}-x^{2} y, v^{2}=a b \subset F$. Also $v=u x+x u$ and $v u=u v=a x$ $+u x u$. Now $u+v=y\left(x^{2}+x\right)-\left(x^{2}+x\right) y$ is a commutator, whence $(u+v)^{2}$ $=d \subset F$ and so $u^{2}+2 u v+v^{2}=d$, and since the characteristic is not 2 , $u v=c \subset F$. Also $u x=x(y x)-(y x) x$ is a commutator and so $u x u x=a e \subset F$. Hence $c=a x+u x u, c x=a x^{2}+a e$, and as $a \neq 0$ we may divide by $a$ and obtain $x^{2}=r x+s, r, s \subset F$.

When $K$ is of characteristic 2 the above proof fails and the proof is more difficult. As before, choose a $y$ which does not permute with $x$ and write $y x+x y=u, u^{2}=a \neq 0, y x^{2}+x^{2} y=v, v^{2}=a b, v=u x+x u$. Again $u v=v u=a x+u x u$ and $u x u x=a e \neq 0$ since $u x$ is a commutator. Here $u v x=a x^{2}+a e$. Clearly $x$ permutes with the right and hence also with the left of this equation. Since $u$ and $v$ permute we may square and obtain $u^{2} v^{2} x^{2}=a^{2} x^{4}+a^{2} e^{2}$ whence $a^{2} b x^{2}$ $=a^{2} x^{4}+a^{2} e^{2}$ and so $x^{4}+b x^{2}+e^{2}=0$ is an equation of fourth degree over $F$ which $x$ satisfies. If $f(x)=x^{4}+b x^{2}+e^{2}$ has a linear factor, then $b$ is a square and $f(x)$ is the square of a polynomial of second degree. Hence if $f(x)$ is reducible over $F$ then $x$ satisfies an equation of second degree over $F$. In particular if the choice of a different $y$ gives different values for $b$ and $e$, then $x$ satisfies an equaticn of second degree over $F$ and the lemma is proved. Hence suppose that $b$ and $e$ depend solely upon $x$ and are independent of $y$. Now $u x u x=a e=a\left(x^{4}+b x^{2}\right) / e$ whence $u x u=a\left(x^{3}+b x\right) / e, u x a=a\left(x^{3}+b x\right) u / e$, $u x=\left(x^{3}+b x\right) u / e$. From this we easily calculate $y x=x y+u, y x^{2}=x y x+u x$ $=x^{2} y+\left[x^{3}+(b+e) x\right] u / e, y x^{3}=x^{3} y+(b+e) u$. Consequently $d=x^{3}+(b+e) x$ permutes with $y$. Since $d$ clearly permutes with every element which permutes with $x$, and also with an arbitrary element $y$ which does not permute with $x$, it follows that $d$ is in the center $F$. As $x$ satisfies an equation of degree lower than the fourth, from earlier remarks $x$ must satisfy an equation of second degree over $F$. This completes the proof of the lemma.

LEMMA 2. If $y z=z y$, then either (1) $1, y, z$ are linearly dependent over $F$ or (2) $F$ has characturistic 2 and $y^{2}=a, z^{2}=b$, with $a, b \subset F$.

Suppose that $1, y, z, y z$ are linearly dependent over $F$. Then either $1, y, z$ are linearly dependent over $F$ or $y z=c y+d z+e$. Here $(y-d) z=c y+e$, $z=(y-d)^{-1}(c y+e)=m y+n$ and in either case $1, y, z$ are dependent over $F$. 
Hence if $1, y, z, y z$ are dependent, so are $1, y, z$.

Let $y^{2}=m y+a, \quad a \neq 0, \quad z^{2}=n z+b, \quad b \neq 0, \quad(y z)^{2}=e y z+f, \quad f \neq 0 . \quad$ Then $(m y+a)(n \Sigma+b)=e y z+f$ and $(m n-e) y z+a n z+b m y+a b-f=0$. Here either $1, y, z, y z$ are dependent and the lemma follows or $m=n=e=0$ and $a b=f$. Now let $(y+z)^{2}=p(y+z)+q$. Here $a+2 y z+b=p y+p z+q$, and if the characteristic is not $2,1, y, z, y z$ are dependent and the lemma follows. If the characteristic is 2 , then $y^{2}=a, z^{2}=b,(y+z)^{2}=a+b$ and no further conclusion may be drawn. This is the second alternative in the lemma.

The remainder of the proof is quite straightforward and will be merely sketched. It is easy to show that if $x$ and $y$ are two elements of $K$ which do not permute then $1, x, y, x y$ are a basis over $F$ for elements generated by $x$ and $y$. As in A. A. Albert $[1$, p. 145] we may suppose $x$ and $y$ so chosen that $x^{2}=x+\beta, x y=y(1-x), y^{2}=\gamma, 4 \beta+1 \neq 0$. Let $z$ be an arbitrary element of $K$. We wish to show that $z$ belongs to the quaternion algebra $Q(1, x, y, x y)$. Replacing $z$ by $z+n x$, if necessary, we have $z^{2}=c$. If $z$ permutes with $x$, by Lemma $2 z$ is dependent on 1 and $x$ and so $z \subset Q$. Suppose then that $z x \neq x z$. From the equation $(x+z)^{2}=p(x+z)+q$ we find that $(p-1)(x z-z x)=0$ whence $p=1$ and so we find that $z x+x z=z+r, r \subset F$. Now putting $z^{\prime}=z+d x+e$ we can determine $d$ and $e$ so that $z^{\prime 2}=c^{\prime}, z^{\prime} x+x z^{\prime}=z^{\prime}$. Now it is easy to verify that $\left(y z^{\prime}\right) x=x\left(y z^{\prime}\right)$ whence, by Lemma $2, y z^{\prime}=s+t x$, since with $x^{2}=x+\beta$ the second alternative cannot arise. Hence $z^{\prime}=y^{-1}(s+t x) \subset Q$ and also $z \subset Q$. Consequently $K=Q$ and we have proved the theorem. Geometrically we have observed that in those planes coordinatized by quaternion algebras there is a seven point configuration theorem, not a consequence of the Desargues Theorem, which corresponds to the universal identity $z(x y-y x)^{2}=(x y-y x)^{2} z$. Conversely we have proved that the assumption of the Desargues Theorem and this additional seven point theorem leads either to the Theorem of Pappus (when $K$ is commutative) or to one of the planes coordinatized by a quaternion algebra.

Although little is known about configuration theorems stronger than the Desargues Theorem, we are somewhat better informed about theorems weaker than the Desargues Theorem. The series of papers by Moufang is concerned with the theorem of the complete quadrilateral, and some special cases of this theorem and the theorems on webs, in particular the Reidemeister figure and the Thomsen triangle as given in the series of papers Topologische Fragen der Differentialgeometrie, are all consequences of the Theorem of Desargues. In fact the author knows of no valid universal theorem which is not at least consistent with the Theorem of Desargues. Theorems exist which are neither stronger than the Theorem of Desargues, nor consequences of it. Theorems of this sort known to the author are those which together with the Desargues Theorem would imply a finite characteristic for the coordinatizing skew-field.

A configuration of considerable interest is given in Figure 4. It is the spe- 
cial case of the Desargues configuration in which the center of perspectivity lies on the axis of perspectivity. The associated configuration theorem is given below.

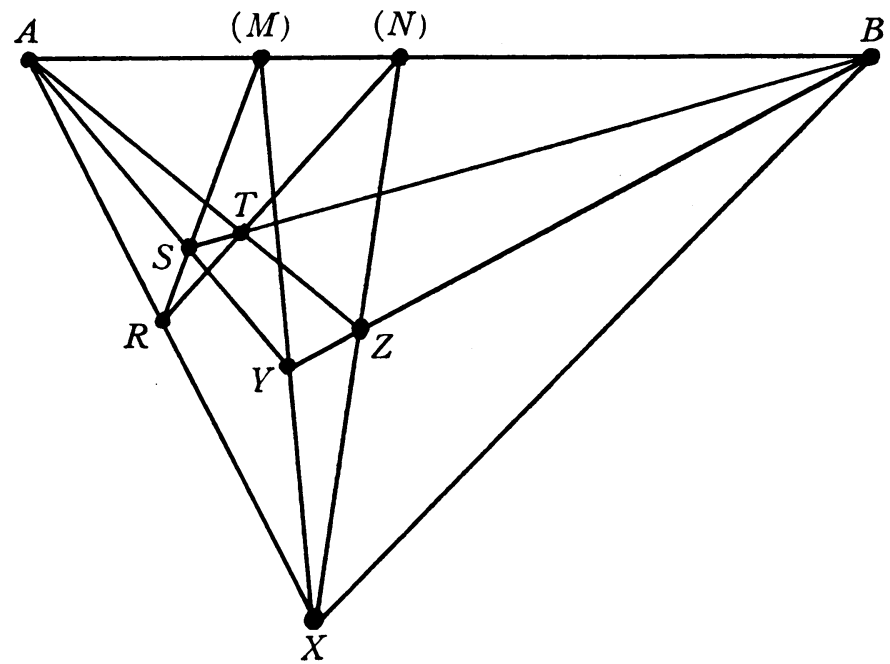

FIG. 4

Theorem L. Given the lines $A M N B, A R X, A S Y, A T Z, R S M, R T N$, $X Y M, X Z N, B Y Z$, then also $B S T$ is a line.

From the same configuration several other equivalent configuration theorems may be formulated. This is clearly a 4 -web theorem as it stands, involving the pencils through $A, B, M=(m)$, and $N=(n)$. Consider the ternary ring with $A, B, O, I$ as the fundamental quadrilateral, where $O I$ intersects $A B$ at $N=(n)=(1)$. The points $X, R$ on $A X$ and $Y$ on $X M$ are arbitrary after $A, B, M$, and $N$ are fixed. Let us take $X=(0,0), R=(0, b), Y=(a, a m)$. Then $S=(a, a \cdot m \circ b), Z=(a m, a m)$, and $T=(a m, a m+b)$. The conclusion that $B S T$ is a line leads to

$$
a \cdot m \circ b=a m+b .
$$

Hence Theorem $\mathrm{L}$ with $A, B, M, N, X$ as fixed points is precisely equivalent to the rule (6.1) which expresses the ternary relation as a combination of two binary relations. Or, otherwise expressed, it is the rule that a line be associated with a linear equation.

Now let us keep $A, B, M, N$ fixed and let $X$ be arbitrary. Write $X=(a, b)$, $R=(a, c)$ where $a, b, c$ are arbitrary. The line $A S Y$ also may be taken arbitrarily as $x=k$. The equations of the lines, using (6.1), will be $y=x+d$ for $X N$ with $b=a+d, y=x m+e$ for $X M$ with $b=a m+e, y=x+f$ for $R N$ with $c=a+f$, and $y=x m+g$ for $R M$ with $c=a m+g$. The points now become 
$Z=(h, h+d), Y=(k, h+d)$ where $h+d=k m+e$ determines $h, T=(h, h+f)$, $S=(k, k m+g)$. The application of the theorem now yields $k m+g=h+f$. The relevant equations are

$$
\begin{gathered}
b=a+d, \quad b=a m+e, \quad c=a+f, \quad c=a m+g, \\
h+d=k m+e, \\
k m+g=h+f,
\end{gathered}
$$

where $a, b, c, k$ are arbitrary, the first five equations determine $d, e, f, g, h$ and the final equation is a consequence of the theorem. Instead of taking $a, b, c, k$ as the arbitrary elements we might equally well take $a, e, g, h$ as the arbitrary elements and consider the system of equations

$$
\begin{aligned}
& a+d=a m+e, \quad a+f=a m+g, \quad h+d=k m+e, \\
& h+f=k m+g \text {, }
\end{aligned}
$$

where the last equation is a consequence of the first three. Here taking $e=0$, $h=0$ we have $a+d=a m, a+f=a m+g, d=k m$, and $f=k m+g=d+g$ whence

$$
a+(d+g)=(a+d)+g
$$

for arbitrary $a$ and $g$, and $d$ determined by $a+d=a m$. This is a restricted associative law of addition. We may use this to prove the general associative law for addition. In (6.3) we may take $d, e, g, h$ as arbitrary since, by (6.1) and property (5) of Theorem 5.4, if $m \neq 1, a+d=a m+e$ determines $a$ uniquely from $d, e$ and $m$. Here take $e=0$. Then $a+d=a m, a+f=a m+g=(a+d)+g$ whence by (6.4) $f=d+g$. Here $h+d=k m$ and by substitution the final equation becomes

$$
(h+d)+g=h+(d+g)
$$

where $h, d, g$ are all arbitrary. Since by Theorem 5.4 there is a zero for addition and in an equation $r+s=t$ any two of $r, s, t$ determine the third uniquely, the associative law (6.5) is all that is needed to prove that addition forms a group. It is readily verified that if addition forms a group, then the last of equations (6.2) is a consequence of the others. Hence Theorem $\mathrm{L}$ for $A, B, M, N=(1)=C$ fixed is equivalent to the linearity (6.1) of the ternary relation and the fact that addition is a group. Note that this does not require the addition to be an abelian group. Note in Figure 4 that permutations of $M, N, B$ do not alter the configuration but that $A$ plays a special role.

Let us apply Theorem $\mathrm{L}$ again with $A$ as the center of the pencil, $y=$ constant; $B$ the center of the pencil, $x=$ constant, $M=(m), N=(1)=C$. If we take $X=(0,0), R=(a, 0)$, and $Z=(b, b)$ with $a, b$ arbitrary we find that $X N$ is $y=x, X M$ is $y=x m, R N$ is $y=x-a, R M$ is $y=x m-a m$. Hence $Y=(b, b m), T=(c, b)$ where $b=c-a$, whence $c=b+a, T=(b+a, b)$. Here $S=(d, b m)$ where $b m=d m-a m$, whence $b m+a m=d m$. By application of 
Theorem L, BST are collinear whence $d=b+a$ and we have

$$
b m+a m=(b+a) m
$$

the right distributive law.

Let us apply Theorem $\mathrm{L}$ again with $M$ as $x=$ constant, $N$ as $y=$ constant, $A=(1), B=(m)$. We may take $X=(0,0), R=(a, a)$ and $A S Y$ as $y=x+b$ where $a$ and $b$ are arbitrary. Then $S=(a, a+b), Y=(0, b)$, whence $B Y Z$ is the line $y=x m+b$ and $Z=(c, 0)$ where $0=c m+b$. Then $A T Z$ is the line $y=x-c$ and since $R T N$ is $y=a, T=(a+c, a)$. By Theorem L, BST are collinear. Let its equation be $y=x m+d$ where $d$ is determined by $a+b=a m+d$. The conclusion is $a=(a+c) m+d$. Hence if $0=c m+b$ and $a+b=a m+d$ then $a=(a+c) m+d$. Instead of considering $a$ and $b$ as the arbitrary elements we may take $b$ and $d$ as the arbitrary elements. Here by (6.6) and the fact that addition forms a group we have $c m=-b,-a m+a=d-b, a=a m+c m+d$, $-a m+a=-b+d$, whence $d-b=-b+d$ and so

$$
b+d=d+b
$$

for arbitrary $b$ and $d$, the commutative law of addition. Hence the natural ring $R$ of the plane $\pi$ satisfies all the conditions of Theorem 5.8 and by Theorem 5.9 this ring is a Veblen-Wedderburn number system with a unit.

THEOREM 6.3. If configuration Theorem $\mathrm{L}$ is satisfied in a plane $\pi$ with $L_{\infty}$ fixed for all choices of $A, B, M, N$ on $L_{\infty}$ with three of these as the center of the pencil $x=$ constant, the center of the pencil $y=$ constant, and the point (1), then $R$, the natural ring determined by a fundamental quadrilateral $A$ (center of $x=$ constant), $B$ (center of $y=$ constant), $O(0,0)$, and $I(1,1)$, is a Veblen-Wedderburn number system with unit. Conversely if $\pi$ is a plane determined by a VeblenWedderburn system, then configuration Theorem $\mathrm{L}$ is valid for all choices of $A, B, M, N$ on $L_{\infty}$.

Proof. In establishing rules (6.1) to (6.7) we have used Theorem $\mathrm{L}$ only with $A, B, M, N$ as including the three points $A, B, C=(1)$ determined by a fundamental quadrilateral $A, B, O, I$. This proves the first part of the theorem.

The converse part of the theorem is stronger than the first part, in that the points $A, B, M, N$ of the configuration, apart from lying on $L_{\infty}$, need bear no special relation to the coordinatizing fundamental quadrilateral $A, B, O, I$. As noted in Theorem 5.9 the translations $(x, y) \rightarrow(x+s, y+t)$ are collineations of $\pi$ leaving the points of $L_{\infty}$ fixed. Hence without loss of generality we may take $X=(0,0)$ in verifying the validity of Theorem L. Suppose $A=(p)$, $B=(q), M=(m), N=(n)$ is a choice of four distinct points on $L_{\infty}$. Here $R=(a, a p)$ with $a$ arbitrary and $Y=(b, b m)$ with $b$ arbitrary. Line equations are as follows: $X R A: y=x p, X Y M: y=x m, X Z N: y=x n, R S M: y=x m-a m$ $+a p, R T N: y=x n-a n+a p, B Y Z: y=x q-b q+b m$ and $A S Y: y=x p-b p$ 
$+b m$. Here if $S=(c, d)$ and $Z=(e, f)$ we have

(6.8) $d=c m-a m+a p, \quad d=c p-b p+b m, \quad f=e n, \quad f=e q-b q+b m$.

From the first two of these we have $(c-a-b) m=(c-a-b) p$ whence, since $m \neq p, c-a-b=0$ and $c=a+b$, whence $d=b m+a p, S=(a+b, b m+a p)$. Here the equation of $A T Z$ is $y=x p-e p+e n$. Hence if $T$ is $(g, h)$ we have $h=g n-a n+a p, h=g p-e p+e n$ and, solving as for $c$ and $d, g=a+e, h=a p+e n$, $T=(a+e, a p+e n)$. It remains to show that $B S T$ are collinear. The equation of $B S$ is $y=x q-a q-b q+b m+a p$. Substituting the coordinates of $T$ we must verify that $a p+e n=(a+e) q-a q-b q+b m+a p$. Here substituting for $e$ from (6.8) we have $a p+e q-b q+b m=a q+e q-a q-b q+b m+a p$, which is a true identity and so $B S T$ are collinear.

Since the center of the pencil $y=$ constant may be represented by (0), this verifies the validity of Theorem $\mathrm{L}$ for all choices of $A, B, M, N$ on $L_{\infty}$ except those including the center of the pencil $x=$ constant as one of the points. This requires two additional verifications, both similar to that given above but somewhat simpler.

This is an affine theorem in that $L_{\infty}$ is fixed but no points on $L_{\infty}$ are fixed. Note that for every choice of fundamental quadrilateral $A, B, O, I$ with $A$ and $B$ on $L_{\infty}$ we get a Veblen-Wedderburn number system, but that these systems need not be algebraically isomorphic. For two different choices of the fundamental quadrilateral it will be found (providing we alter the coordinates suitably) that addition is the same but that one multiplication may be found from the other by a rule on the order of (5.12), which is the special rule when $A_{1}=A_{2}, B_{1}=B_{2}$. In appendix II are examples of non-isomorphic systems defining the same plane. The number of non-isomorphic systems is clearly connected with the nature of the collineation group of the plane. The collineation group need not be doubly transitive on the points of $L_{\infty}$, but in these examples and others not here given the group is simply transitive on the points of $L_{\infty}$. It would be interesting to investigate the collineation groups of these planes and the geometric equivalence of the Veblen-Wedderburn systems more thoroughly.

Now let us consider those planes in which Theorem $\mathrm{L}$ is a universal theorem, permitting the line $A B M N$ to be arbitrary. Naturally the laws holding for the affine theorem will also hold for the universal theorem. Let us first take $A=A, B=O, M=(0, a), N=(0, b), X=B, R=(m)$ and let the line $A S Y$ be $x=c$. Here $a \neq 0, b \neq 0, a \neq b, c \neq 0$, and $m \neq 0$ are arbitrary. Here the equations of the lines are RSM:y=xm+a, RTN:y=xm+b, MYX:y=a,NZX: $y=b$. Here if $Y=(c, a)$ and $a=c r, B Y Z$ is $y=x r$, whence $Z=(d, b)$ with $b=d r$. Then $S$ as the intersection of $A S Y$ and $R S M$ is $S=(c, c m+a)$, while $T$ as the intersection of $A T Z$ and $R T N$ is $T=(d, d m+b)$. The theorem then states that $B S T$ are collinear and so if $c m+a=c s$ determines $s$, then $d m+b$ $=d s$. Take $c, d, r, m$ instead of $a, b, c, m$ as the arbitrary elements. The 
theorem becomes: if $c m+c r=c s(c \neq 0)$, then $d m+d r=d s$. Putting $c=1$ we have $m+r=s$, whence

$$
d m+d r=d(m+r),
$$

the left distributive law. Clearly this law is a consequence of the left distributive law and so is equivalent to it. The two distributive laws have as immediate consequences

$$
-a m=(-a) m=a(-m) .
$$

Finally let us apply Theorem L with $A=(0,0), B=B, M=(a, 0)$, $N=(b, 0), X=A, R=(0, c)$ and $Y=(a, a m)$ where $a \neq 0, b \neq 0, a \neq b, c \neq 0$, $m \neq 0$ are arbitrary. Here $Z=(b, a m)$. Line equations will be: $A B M N: y=0$. $A R X: x=0, A S Y: y=x m, A T Z: y=x n$ where $a m=b n, M S R: y=x p+c$ where $0=a p+c, M X Y: x=a, N R T: y=x q+c$ where $0=b q+c, N X Z: x=b, B Y Z:$ $y=a m$. We now find $S=(d, d m)$ where $d m=d p+c$ and $T=(e$, en $)$ where $e n=e q+c$. The theorem that $B S T$ are collinear yields $e n=d m$. The relevant equations are

$$
\begin{aligned}
& a m=b n, \quad 0=a p+c, \quad 0=b q+c, \\
& d m=d p+c, \quad e n=e q+c, \quad e n=d m .
\end{aligned}
$$

We may eliminate $c$ from these equations and obtain

$$
a m=b n, \quad a p=b q, \quad a p=d p-d m, \quad a p=e q-e n, \quad e n=d m,
$$

where $a, b, m, p$ are arbitrary and the last equation is a consequence of the first four. If we put $a=1, p=1, m=1-b$, we find that $b q=1, d b=1, n=q-1$, $e=1$, and finally $q=d$, whence $b q=q b=1$ and we may write $q=b^{-1}$,

$$
b b^{-1}=b^{-1} b=1,
$$

stating that the right and left inverses of an element are equal. (The excluded possibility $m=0$ corresponds to $b=1$, where clearly $b^{-1}=1$.) Now put $a=1, p=1, m=1-b r$ where $b$ and $r$ are arbitrary. This leads to $q=b^{-1}$, $n=b^{-1}-r, d=(b r)^{-1}, e=r^{-1}$ and finally

$$
(b r)^{-1}=r^{-1} b^{-1} \text {. }
$$

Here the excluded possibility $m=0$ corresponds to $b r=1$ which is covered by (6.13).

Again let us put $m=a^{-1}$ and choose $p$ so that $a p=b q=q-b^{-1}$. We have immediately $n=b^{-1}, a p=e\left(q-b^{-1}\right)$, whence $e=1$. The last equation becomes $b^{-1}=d a^{-1}$ and combined with $a p=q-b^{-1}=d p-d a^{-1}$ leads to $q=d p$. The system of equations now reduces to

$$
a p=q-b^{-1}, \quad a p=b q, \quad q=d p, \quad b^{-1}=d a^{-1} .
$$


Here $b(a p)=b\left(q-b^{-1}\right)=b q-1=a p-1$ whence $(1-b)(a p)=1, a p=(1-\dot{b})^{-1}$ and $a p-1=(1-b)^{-1}-1=(1-b)^{-1}(1-1+b)=(1-b)^{-1} b=\left[b^{-1}(1-b)\right]^{-1}$ $=\left(b^{-1}-1\right)^{-1}$. Also $d p-d a^{-1}=q-b^{-1}=a p=(1-b)^{-1}$ and

$$
\begin{aligned}
d\left(p-a^{-1}\right) & =(1-b)^{-1}, \\
d\left[a^{-1}(a p-1)\right] & =(1-b)^{-1}, \\
d\left[a^{-1}\left(b^{-1}-1\right)^{-1}\right] & =(1-b)^{-1}, \\
d\left[\left(b^{-1}-1\right) a\right]^{-1} & =(1-b)^{-1}, \\
{\left[\left(b^{-1}-1\right) a\right] d^{-1} } & =1-b, \\
\left(b^{-1} a-a\right) d^{-1} & =1-b, \\
\left(b^{-1} a\right) d^{-1}-a d^{-1} & =1-b .
\end{aligned}
$$

But $a d^{-1}=b$ by (6.14) and (6.15) whence $\left(b^{-1} a\right) d^{-1}=1$, and so $b^{-1}=\left(b^{-1} a\right) a^{-1}$. By (6.14) we have also $b=\left[\left(b^{-1} a\right) a^{-1}\right]^{-1}=a\left(a^{-1} b\right)$. We write these two restricted associative laws in the form

$$
a^{-1}(a b)=b=(b a) a^{-1} .
$$

It is quite easy to show that (6.14) is a consequence of (6.13) and (6.16). A Veblen-Wedderburn system satisfying also the left distributive law (6.9), the law of unique inverse (6.13), and the weak associative laws (6.16) is an alternative field $\left({ }^{18}\right)$. The reader may find it an interesting exercise of ingenuity to show that from these laws the last of equations (6.12) is a consequence of the first four, but it is not necessary for our purposes to do so.

Theorem 6.4(19). If Theorem $\mathrm{L}$ is satisfied for all choices of $A, B, M, N$ on two lines in a plane $\pi$, then Theorem $\mathrm{L}$ is a universal theorem in $\pi$ and any natural ring associated with $\pi$ satisfies

$$
\begin{array}{ll}
a \cdot m \circ b=a m+b, & a(b+c)=a b+a c, \\
(a+b)+c=a+(b+c), & (a+b) m=a m+b m, \\
a+b=b+a, & a 1=1 a=a, \\
a+0=0+a=a, & a a^{-1}=a^{-1} a=1, \quad a \neq 0, \\
a+(-a)=(-a)+a=0, & a^{-1}(a b)=b=(b a) a^{-1}, \quad a \neq 0,
\end{array}
$$

(18) R. Moufang $[6,7]$, M. Zorn $[1,2]$.

(19) R. Moufang derives alternative fields from the theorem of the complete quadrilateral, most of the geometric part of her proof being in her third paper. Her Theorem 3a [Moufang 3, p. 762] is essentially the same as Theorem $\mathrm{L}$ in this paper. But her derivation assumes order and her method of proof is certainly not valid if the diagonal points of every quadrilateral are collinear. (This is essentially the geometric configuration corresponding to characteristic in the coordinatizing ring.) Curiously. enough she does not seem to have observed that her Theorem 2, after renumbering vertices, is identical with her Theorem $D_{9}$ (Theorem 1 ), but gives proofs of their equivalence. 
and is an alternative field. The collineation group of $\pi$ is transitive on the triangles of $\pi$.

Proof. We have shown that if Theorem $\mathrm{L}$ is satisfied for all choices of $A, B, M, N$ on $L_{\infty}$ and on $x=0$, then rules (6.17) are valid for the natural ring $R$ determined by the quadrilateral $A, B, O, I$. In $\pi$ there exist the following collineations:

( $\alpha$ ) Translations:

$$
\begin{aligned}
(x, y) & \rightarrow(x+r, y+s), \\
A & \rightarrow A, \\
(m) & \rightarrow(m), \\
x=c & \rightarrow x=c+r, \\
y=x m+b & \rightarrow y=x m+(r m+b-s) .
\end{aligned}
$$

( $\beta$ ) The reflection:

$$
\begin{aligned}
(x, y) & \rightleftarrows(y, x), & \\
A & \rightleftarrows B, & \\
(m) & \rightleftarrows\left(m^{-1}\right) & (m \neq 0), \\
x=c & \rightleftarrows y=c, & \\
y=x m+b & \rightleftarrows y=x m^{-1}-b m^{-1} & (m \neq 0) .
\end{aligned}
$$

$(\gamma)$

$$
\begin{aligned}
A & \rightarrow A, \\
(m) & \rightarrow(m+r), \\
(x, y) & \rightarrow(x, y+x r), \\
x=c & \rightarrow x=c, \\
y=x m+b & \rightarrow y=x(m+r)+b .
\end{aligned}
$$

( $\delta$ ) The reflection:

$$
\begin{array}{rlr}
A & \rightleftarrows A, & \\
(m) & \rightleftarrows(0, m), & \\
(x, y) & \rightleftarrows\left(x^{-1}, x^{-1} y\right) & (x \neq 0), \\
x=0 & \rightleftarrows L_{\infty}, & \\
x=a & \rightleftarrows x=a^{-1} & (a \neq 0), \\
y=x m+b & \rightleftarrows y=x b+m .
\end{array}
$$

The validity of $(\alpha)$ has already been noted in Theorem 5.9. $(\beta)$ is easily verified by observing that if $v=u m+b, v m^{-1}=(u m) m^{-1}+b m^{-1}=u+b m^{-1}$, and 
$u=v m^{-1}-b m^{-1}$. For $(\gamma)$ we observe that if $y=x m+b$, then by the left distributive law $y+x r=x(m+r)+b$. For $(\delta)$ the verifications are trivial except to show that from $v=u m+b, u \neq 0, u^{-1} v=u^{-1}(u m)+u^{-1} b=u^{-1} b+m$.

The collineation $(\delta)$ takes $L_{\infty}$ into $x=0 .(\alpha)$ takes $x=0$ into $x=r$, an arbitrary line through $A$ different from $L_{\infty}$. $(\beta)$ takes the pencil through $A$ into the pencil through $B .(\gamma)$ takes the pencil through $B$ into the pencil through an arbitrary $(r)$. Hence the collineation group of $\pi$ is transitive on the lines of $\pi$, and an arbitrary line may be taken into $L_{\infty}$. Hence since Theorem $\mathrm{L}$ is valid for all choices of $A, B, M, N$ on $L_{\infty}$, by Theorem 6.3 , it is a universal theorem. Hence for an arbitrary choice of the fundamental quadrilateral, Theorem $\mathrm{L}$ will be satisfied for $A B M N$ on the new $L_{\infty}$ and the new $x=0$ and so the natural ring will satisfy the rules (6.17).

Consider any triangle $P Q S$ of $\pi$. By a collineation we may take the line $P Q$ into $L_{\infty}$. By a combination of $(\gamma)$, which leaves $A$ fixed and is transitive on the remaining points of $L$, and $(\beta)$, which interchanges $A$ and $B$, we may take two arbitrary points of $L_{\infty}$ into $A$ and $B$. Then $(\alpha)$ leaves $A$ and $B$ fixed and takes an arbitrary point not on $L_{\infty}$ into $O(0,0)$. Hence there is a collineation taking $P Q S$ into $A B O$ and the collineations of $\pi$ are transitive on the triangles of $\pi$.

It is not known to the author whether or not the collineations of $\pi$ are transitive on quadrilaterals. If they are, an arbitrary choice of the fundamental quadrilateral will yield the same alternative field as the natural ring for $\pi$. If they are not, every natural ring will be an alternative field, but nonisomorphic alternative fields will determine the same plane. One may be obtained from another by the rule (5.12). It may be shown that the collineations of $\pi$ will be transitive on quadrilaterals if and only if for every $r \neq 0$ there exists a function $\phi_{r}(u)$ such that

$$
\phi_{r}(a)+\phi_{r}(b)=\phi_{r}(a+b), \quad \phi_{r}(a m)=\phi_{r}(a)\left[r^{-1} \phi_{r}(m)\right] .
$$

Obviously, when the multiplication is associative $\phi_{r}(u)=r u$ satisfies these conditions.

Theorem $\mathrm{L}$ is a special case of the Theorem of Desargues. If we assume the Theorem of Desargues as a universal theorem, then not only the rules (6.17) must be valid for the natural rings associated with the plane, but, taking the origin as the center of perspectivity and $L_{\infty}$ as the axis of perspectivity, it is easily shown that multiplication is associative, and that $R$ is a skew-field. Then the homogeneous representation of $\pi$ is easily established and the collineations (5.2) show that every Desarguesian plane is determined by a unique skew-field, and hence is an analytic plane. Conversely the verification of the validity of the Desargues Theorem in an analytic plane is a straightforward and relatively simple calculation. But to prove that every Desarguesian plane is an affine plane it is simpler to rely on the methods of standard texts than to use Theorem 6.3 which depends on the weaker Theorem L. 


\section{APPENDIX I}

THEOREM. Given permutations $S$ on a finite number $n$ of letters such that (a) $S$ is doubly transitive, (b) if $M, L \subset S$ and $M \neq L$, then $M L^{-1}$ fixes at most one letter, then there are $n-1$ permutations taking a given letter $i$ into a given letter $j$ and (c) if $L$ does not take $i$ into $j$ there is exactly one $M$ taking $i$ into $j$ such that $M L^{-1}$ displaces all letters.

Denote the letters by $1,2, \cdots, n$. Consider the permutations $T(i, j)$ $=(\cdots, i, k, \cdots)$ for fixed $i, j$, and $k \neq i$. $a_{k}$ may take any value $1, \cdots, n$ except $j$. Hence there are at least $n-1$ permutations $T(i, j)$ since $S$ is doubly transitive. If two $T(i, j)$ permutations $A, B$ have the same value for $a_{k}$, then $A B^{-1}$ fixes both $i$ and $k$ and by condition (b) $A=B$. Hence there are exactly $n-1$ permutations $T(i, j)$. Suppose $L$ does not take $i$ into $j$,

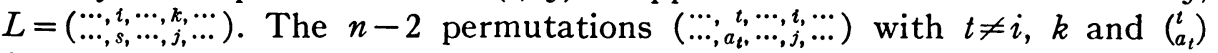
from $L$ are different from $L$ and each other, since $L$ does not take $i$ into $j$, and if two of these were the same $N$ then $N L^{-1}$ would fix two letters. Call the remaining $T(i, j), M$. Then $M$ takes $i$ into $j$ and $M L^{-1}$ displaces all letters.

If $S$ is a set of permutations on infinitely many letters satisfying (a) and (b) then (c) may be violated in either of two ways. Either for a given $L$ and $\left(\begin{array}{l}i \\ j\end{array}\right)$ there may be no $M$ taking $i$ into $j$ such that $M L^{-1}$ displaces all letters or there may be more than one. Both possibilities actually arise. Consider permutations on the non-negative integers $0,1,2, \ldots$. Suppose given a finite set $P_{1}=\left(\begin{array}{l}a_{11}, \cdots, a_{11}, \cdots \\ b_{11}, \cdots, b_{1 r}, \cdots\end{array}\right), \cdots, P_{i}=\left(\begin{array}{l}a_{i 1}, \ldots \\ b_{i 1}, \ldots\end{array}\right), \cdots, P_{s}=\left(\begin{array}{l}a_{s 1}, \cdots \\ b_{s 1}, \cdots\end{array}\right)$ where the $a$ 's and $b$ 's are subject to the following conditions: (A) $(\cdots, c, \cdots, \cdots, \cdots, \cdots), c \neq e, d \neq f$, occurs in at most one $P$ for any combination of $c, d, e, f$ and every combination with $c+d+e+f \leqq N$ does occur; and (B) For each $P_{i}, a_{i 1}, \ldots \ldots$ are distinct and include $1,2, \cdots, N$ and $b_{i 1}, \cdots$ are distinct and include $1,2, \cdots, N$. Then it is possible to extend $P_{1}, \cdots, P_{s}$ for $N=k$ to a larger system $P_{1}, \cdots, P_{t}$ which satisfies (A) and (B) for $N=k+1$. In a countable number of steps we have then constructed a set $S$ of permutations satisfying (a) and (b). If all $(\cdots, c, \cdots, \cdots, \cdots, \cdots, \ldots)$ with $c+d+e+f=k+1$ occur in $P_{1}, \cdots, P_{s}$ then no extension is needed to satisfy (A) for $N=k+1$. If one such combination has not occurred write $P_{s+1}=\left(\begin{array}{c}c, e, \\ d, f\end{array}\right)$ and with a finite number of such adjunctions (A) is satisfied for $N=k+1$. Now if a given $i \leqq k+1$ has not occurred in some $P$ as an $a$, adjoin to this $P$ the combination $\left(\begin{array}{l}i \\ n\end{array}\right)$ where if $n$ is sufficiently large (A) will not be violated, since $n$ must satisfy only a finite number of inequalities. Similarly adjoin $\left(\begin{array}{l}n \\ i\end{array}\right)$ to any $P$ in which $i \leqq k+1$ has not occurred as a $b$. If $n$ is large enough this may be done without violating (A). Hence in a finite number of steps we may extend $P_{1}, \cdots, P_{s}$, satisfying (A) and (B) for $N=k$, to $P_{1}, \cdots, P_{t}$ satisfying (A) and (B) for $N=k+1$. Since we do not alter the letters originally in the $P$ 's but merely adjoin new letters and new $P$ 's there is no ambiguity in considering the result of a countable number 
of steps, and this is a set $S$ satisfying (a) and (b).

If we start with

$$
P_{1}=\left(\begin{array}{l}
0,1,2,3,4, \cdots, n, \cdots \\
0,1,2,3,4, \cdots, n, \cdots
\end{array}\right), \quad P_{2}=\left(\begin{array}{l}
0,1,2,3,4, \cdots, 2 r-1, \\
1,2,0,4,3, \cdots, 2 r, \ldots
\end{array} 2 r, 2 r-1, \cdots\right), \quad P_{3}=\left(\begin{array}{l}
0,1,2,3, \cdots, 2 \\
1,0,3,2, \cdots, 2 r+1,2 r+1, \cdots
\end{array} 2 r, \cdots\right),
$$

we have (A) and (B) satisfied for $k=2$. As $k \rightarrow \infty, P_{1}, P_{2}, P_{3}$ remain unchanged, and the complete set $S$ satisfies (a) and (b). But here both $P_{2}$ and $P_{3}$ take 0 into 1 and $P_{2} P_{1}^{-1}$ and $P_{3} P_{1}^{-1}$ displace all letters, thus violating (c). On the other hand if we start with $P_{1}=\left(\begin{array}{l}0,1,2, \cdots, n, \ldots \\ 0,1,2, \cdots, n, \ldots\end{array}\right), P_{2}=\left(\begin{array}{l}0,1,2 \\ 0,1,2\end{array}\right)$ we have (A) and (B) satisfied for $k=2$. At each stage if some $P_{i}$ does not fix any letter we may adjoin $\left(\begin{array}{l}c \\ c\end{array}\right)$ to $P_{i}$ with a sufficiently large $c$. In this case there is no $P$ such that $P P_{1}^{-1}$ displaces all letters and (c) is violated the other way.

\section{APPENDIX II}

Of the Veblen-Wedderburn systems with nine elements, there are, besides the finite field, four non-isomorphic systems. In all four the additive group is the elementary abelian group of order 9 and the elements may be represented as $0,1,2, a, a+1, a+2,2 a, 2 a+1,2 a+2$, where we add elements $i a+j$ by taking $i$ and $j$ modulo 3 . The multiplication tables of the four systems are given below. Since $0 x=x 0=0,0$ is omitted from the table. The product $u v$ is in the row beginning with $u$ and in the column under $v$. The element 2 is associative on the left since $(2 x) y=(x+x) y=x y+x y=2(x y)$, but need not be in the center (System $U$ ).

\begin{tabular}{|c|c|c|c|c|c|c|c|}
\hline 1 & 2 & $a$ & $a+1$ & $a+2$ & $2 a$ & $2 a+1$ & $2 a+2$ \\
\hline 2 & 1 & $2 a$ & $2 a+2$ & $2 a+1$ & $a$ & $a+2$ & $a+1$ \\
\hline$a$ & $2 a$ & 2 & $2 a+1$ & $a+1$ & 1 & $2 a+2$ & $a+2$ \\
\hline$a+1$ & $2 a+2$ & $a+2$ & 2 & $2 a$ & $2 a+1$ & $a$ & 1 \\
\hline$a+2$ & $2 a+1$ & $2 a+2$ & $a$ & 2 & $a+1$ & 1 & $2 a$ \\
\hline $2 a$ & $a$ & 1 & $a+2$ & $2 a+2$ & 2 & $a+1$ & $2 a+1$ \\
\hline $2 a+1$ & $a+2$ & $a+1$ & $2 a$ & 1 & $2 a+2$ & 2 & $a$ \\
\hline $2 a+2$ & $a+1$ & $2 a+1$ & 1 & $a$ & $a+2$ & $2 a$ & 2 \\
\hline \multicolumn{8}{|c|}{ S. $\quad z^{2}=z+1$} \\
\hline 1 & 2 & $a$ & $a+1$ & $a+2$ & $2 a$ & $2 a+1$ & $2 a+2$ \\
\hline 2 & 1 & $2 a$ & $2 a+2$ & $2 a+1$ & $a$ & $a+2$ & $a+1$ \\
\hline$a$ & $2 a$ & $a+1$ & 1 & $2 a+2$ & $a+2$ & 2 & $2 a+1$ \\
\hline$a+1$ & $2 a+2$ & $2 a+1$ & $a+2$ & 1 & 2 & $2 a$ & $a$ \\
\hline$a+2$ & $2 a+1$ & 1 & $2 a$ & $a$ & $2 a+2$ & $a+1$ & 2 \\
\hline $2 a$ & $a$ & $2 a+2$ & 2 & $a+1$ & $2 a+1$ & 1 & $a+2$ \\
\hline $2 a+1$ & $a+2$ & 2 & $a$ & $2 a$ & $a+1$ & $2 a+2$ & 1 \\
\hline $2 a+2$ & $a+1$ & $a+2$ & $2 a+1$ & 2 & 1 & $a$ & $2 a$ \\
\hline
\end{tabular}




\begin{tabular}{cccccccc}
\multicolumn{7}{c}{$T . z^{2}=2 z+1$} \\
1 & 2 & $a$ & $a+1$ & $a+2$ & $2 a$ & $2 a+1$ & $2 a+2$ \\
2 & 1 & $2 a$ & $2 a+2$ & $2 a+1$ & $a$ & $a+2$ & $a+1$ \\
$a$ & $2 a$ & $2 a+1$ & $a+2$ & 1 & $2 a+2$ & $a+1$ & 2 \\
$a+1$ & $2 a+2$ & 1 & $2 a$ & $a$ & $a+2$ & 2 & $2 a+1$ \\
$a+2$ & $2 a+1$ & $a+1$ & 1 & $2 a+2$ & 2 & $2 a$ & $a$ \\
$2 a$ & $a$ & $a+2$ & $2 a+1$ & 2 & $a+1$ & $2 a+2$ & 1 \\
$2 a+1$ & $a+2$ & $2 a+2$ & 2 & $a+1$ & 1 & $a$ & $2 a$ \\
$2 a+2$ & $a+1$ & 2 & $a$ & $2 a$ & $2 a+1$ & 1 & $a+2$
\end{tabular}

U. 2 not in center

$\begin{array}{cccccccc}1 & 2 & a & a+1 & a+2 & 2 a & 2 a+1 & 2 a+2 \\ 2 & 1 & 2 a & 2 a+2 & 2 a+1 & a & a+2 & a+1 \\ a & 2 a+1 & a+2 & 1 & 2 a & a+1 & 2 a+2 & 2 \\ a+1 & 2 a & 2 a+2 & a+2 & 2 & 1 & a & 2 a+1 \\ a+2 & 2 a+2 & 2 & 2 a & a+1 & 2 a+1 & 1 & a \\ 2 a & a+2 & 2 a+1 & 2 & a & 2 a+2 & a+1 & 1 \\ 2 a+1 & a+1 & 1 & a & 2 a+2 & a+2 & 2 & 2 a \\ 2 a+2 & a & a+1 & 2 a+1 & 1 & 2 & 2 a & a+2\end{array}$

System $R$ is a group system which may be derived from a doubly transitive group on 9 letters in which only the identity fixes two letters, as in Theorems 5.6 and 5.7. The subgroup of elements fixing one letter (the multiplicative group of the Veblen-Wedderburn system) is the quaternion group.

In systems $S$ and $T$ the elements $0,1,2$ are in the center and in $S$ every other element $z$ satisfies $z^{2}=z+1$, while in $T$ every other element $z$ satisfies $z^{2}=2 z+1$. In system $U$ the center consists solely of the elements 0,1 . In $S, T, U$ it is immediately observed that $(a a) a \neq a(a a)$.

Systems $S$ and $T$ suggest a method of constructing Veblen-Wedderburn systems $J$ which turns out to be valid. Given a commutative field $F$ and a polynomial with coefficients from $F, z^{2}-r z-s$, irreducible over $F$. Let the elements of $J$ be $a u+b, a, b \subset F$, and let addition in $J$ be defined by $(a u+b)$ $+(c u+d)=(a+c) u+(b+d)$. For multiplication let $c(a u+b)=(a u+b) c=a c u$ $+b c$ and $c(w v)=(c w) v$ if $a, b, c \subset F$. Also let $z^{2}=r z+s$ if $z=a u+b, a \neq 0$. These rules, together with the right distributive law, completely determine the multiplication table for $J$. It may be verified with a certain amount of tedious calculation that $J$ then satisfies all requirements of Theorem 5.8 and is a Veblen-Wedderburn system. $J$ will not be a field or even a skew-field unless $F$ is the finite field with two elements.

Although the four systems $R, S, T, U$ are not algebraically isomorphic, they all define the same projective plane. Systems $S, T, U$ define planes $\pi(S), \pi(T), \pi(U)$ isomorphic to each other with $A \rightarrow A, B \rightarrow B$, and each may be obtained from the other by an appropriate application of rule (5.12). To 
map $\pi(T)$ on to $\pi(S)$ take $a=1, s=2$ in (5.12). $\pi(T) \rightarrow \pi(S),(x, 2 y) \rightarrow(x, y)$. The equation $(x \circ d) 2=x(d 2)$ defines a product $x \circ d$ in $S$ in terms of the multiplication in $T$. To map $\pi(U)$ onto $\pi(S)$, take $a=1, s=2 a+2$ in (5.12). $\pi(U) \rightarrow \pi(S),[x, y(2 a+2)] \rightarrow[x, y]$. The equation $(x \circ d)(2 a+2)=x[d(2 a+2)]$ defines a product $x \circ d$ in $S$ in terms of the multiplication in $U$. There is no isomorphism between $\pi(R)$ and $\pi(S)$ leaving $A$ and $B$ fixed. To map $\pi(S)$ onto $\pi(R)$ let us take the quadrilateral $A,(2),(0,0),(1,0)$ in $\pi(S)$ and use it as a fundamental quadrilateral to coordinatize the plane. By Theorem 6.3 this will lead to a Veblen-Wedderburn system and the lines in the plane will be determined by equations $x=c, y=x m+b$. Keeping $x$ coordinates unchanged this leads to $(x, y) \rightarrow(x, x+y),(m) \rightarrow(1+m)$. In the new ring addition is found to be the same as in $S$, and multiplication $u \circ m$ is given by the rule $u \circ m=u(m-1)+u$. The new ring is the system $R$ and so we have mapped $\pi(S)$ onto $\pi(R)$. Hence we have shown that all four systems define the same plane $\pi$.

What is the collineation group of $\pi$ ? If there were a collineation of $\pi$ taking $L_{\infty}$ into another line then Theorem $\mathrm{L}$ would be satisfied for all $A, B, M, N$ on two lines, and hence by Theorem 6.4 every natural ring of $\pi$ would be an alternative field. Since this is not the case, the collineations of $\pi$ leave $L_{\infty}$ fixed. Taking $\pi$ as represented by $R$, the following mappings of points are collineations:

(1)

$$
\begin{aligned}
A & \rightarrow(2), \\
B=(0) & \rightarrow(1), \\
(1) & \rightarrow(0), \\
(2) & \rightarrow A, \\
(m) & \rightarrow(2 m) \\
(x, y) & \rightarrow(x+y, x-y), \\
A & \rightarrow A, \\
(m) & \rightarrow\left(r^{-1} m s\right) \\
(x, y) & \rightarrow(x r, y s), \\
A & \rightarrow A, \\
(m) & \rightarrow(m), \\
(x, y) & \rightarrow(x+r, y+s) \quad(r, s \text { fixed parameters } \neq 0),
\end{aligned}
$$

These generate the complete collineation group of $\pi$ which is of order $10 \cdot 8 \cdot 6 \cdot 4 \cdot 2 \cdot 81=311,040$. The collineations induce a transitive permutation group on the points of $L_{\infty}$ of order $10 \cdot 8 \cdot 6 \cdot 4 \cdot 2=3840$. In the symmetric group on the ten points of $L_{\infty}, A,(0),(1),(2),(a),(a+1),(a+2),(2 a),(2 a+1)$, 
$(2 a+2)$, this group is the centralizer of the element $[A,(0)][(1),(2)]$ $[(a),(2 a)][(a+1),(2 a+2)][(a+2),(2 a+1)]$.

The full projective group of $\pi$ is the symmetric group on the points of a line, and hence is of relatively little interest.

\section{BibLIOGRAPHY}

\section{A. A. Albert}

1. Structure of algebras, Amer. Math. Soc. Colloquium Publications, vol. 24.

GARRETT BIRKHOFF

1. Combinatorial relations in projective geometries, Ann. of Math. vol. 36 (1935) pp. 743-748.

W. BLASCHKE AND G. BoL

1. Geometrie der Gewebe, Berlin, Springer, 1938.

R. D. Carmichael

1. Groups of finite order, Ginn and Co., 1937.

E. W. Davis

1. A geometric picture of the fifteen schoolgirls problem, Ann. of Math. vol. 2 pp. 156-157.

O. ECKENSTEIN

1. Bibliography of Kirkman's schoolgirl problem, Messenger of Mathematics vol. 41 (1911)

А. Емсн pp. 33-36.

1. Triple and multiple systems, their geometric configurations and groups, Trans. Amer. Math. Soc. vol. 31 (1929) pp. 25-42.

G. HeSSENBERG

1. Beweis des Desarguesschen Satzes aus dem Pascalschen, Math. Ann. vol. 61 (1905).

D. HILBERT

1. Grundlagen der Geometrie, Teubner, 7th edition, 1930.

P. Jordan, J. von NeumanN, AND E. Wigner

1. On an algebraic generalization of the quantum mechanical formalism, Ann. of Math. (2) vol. 35 (1934) pp. 29-64.

T. P. KIRKMAN

1. Schoolgirl problem, The Lady's and Gentleman's Diary 1850.

A. KuROSCH

1. Ringtheoretische Probleme, die mit dem Burnsideschen Problem über periodische Gruppen F. LEVI in Zusammenhang stehen, Bull. Acad. Sci. URSS. Sér. Math. vol. 5 (1941) pp. 233-240.

1. Geometrische Konfigurationen, Hirzel, Leipzig, 1929.

D. E. LitTlewood

1. Identical relations satisfied in an algebra, Proc. London Math. Soc. (2) vol. 32 (1931) pp. 312-320.

K. MENGER

1. Bemerkungen zu Grundlagenfragen. IV, Jber. Deutschen Math. Verein vol. 37 (1928) pp. 309-313.

2. New foundations of projective and affine geometry, Ann. of Math. (2) vol. 37 (1936) pp. 456-482.

Ruth Moufang

1. Zur Struktur der projektiven Geometrie der Ebene, Math. Ann. vol. 105 (1931) pp. 536601.

2. Die Einfuhrung der idealen Elemente in die ebene Geometrie mit Hilfe des Satzes vom vollstandigen Vierseit, Math. Ann. vol. 105 (1931) pp. 759-778.

3. Die Schnittpunktsatze des projektiven speziellen Funfecksnetzes in ihrer Abhangigkeit voneinander. (Das A-Netz), Math. Ann. vol. 106 (1932) pp. 755-795. 
4. Ein Satz über die Schnittpunktsatze des allgemeinen Funfecksnetzes [Das $(A, B)-N e t z]$, Math. Ann. vol. 107 (1932) pp. 124-139.

5. Die Desarguesschen Satz vom Rang 10, Math. Ann. vol. 108 (1933) pp. 296-310.

6. Alternativkörper und der Satz vom vollstandigen Vierseit $\left(D_{9}\right)$, Abh. Math. Sem. Hamburgischen Univ. vol. 9 (1933) pp. 207-222.

7. Zur Struktur von Alternativkörpern, Math. Ann. vol. 110 (1934) pp. 416-430.

B. H. NeumanN

1. Identical relations in groups. I, Math. Ann. vol. 114 (1937) pp. 506-525.

2. On the commutativity of addition, J. London Math. Soc. vol. 15 (1940) pp. 203-208.

A. R. RICHARDSON

1. Equations over a division algebra, Messenger of Mathematics vol. 57 (1928) pp. 1-6. A. SPEISER

1. Theorie der Gruppen von endlicher Ordnung, 2nd edition (1927), J. Springer, Berlin.

K. G. C. von Staudt

1. Beitrage zur Geometrie der Lage, vol. 2, 1857.

G. THOMSEN

1. Grundlagen der Elementargeometrie, Leipzig, 1933.

O. VEBLEN AND J. H. M. WEDdERBURN

1. Non-Desarguesian and non-Pascalian geometries, Trans. Amer. Math. Soc. vol. 8 (1907) pp. 379-388.

O. Veblen AND J. W. Young

1. Projective geometry, vol. I, Ginn and Co., 1910.

W. WAGNER

1. Über die Grundlagen der projektiven Geometrie und allgemeine Zahlensysteme, Math. Ann. vol. 113 (1936-1937) pp. 528-567.

M. ZORN

1. Theorie der alternativen Ringe, Abh. Math. Sem. Hamburgischen Univ. vol. 8 (1930) pp. 123-147.

2. Alternativkörper und quadratische Systeme, Abh. Math. Sem. Hamburgischen Univ. vol. 9 (1933) pp. 395-402.

YALE UNIVERSITY, New Haven, Conn. 